\title{
PRIMER
}

\section{Invasive candidiasis}

\section{Peter G. Pappas ${ }^{1}$, Michail S. Lionakis², Maiken Cavling Arendrup $3,4,5$, Luis Ostrosky-Zeichner ${ }^{6}$ and Bart Jan Kullberg ${ }^{7}$}

Abstract / Invasive candidiasis is an important health-care-associated fungal infection that can be caused by several Candida spp.; the most common species is Candida albicans, but the prevalence of these organisms varies considerably depending on geographical location. The spectrum of disease of invasive candidiasis ranges from minimally symptomatic candidaemia to fulminant sepsis with an associated mortality exceeding 70\%. Candida spp. are common commensal organisms in the skin and gut microbiota, and disruptions in the cutaneous and gastrointestinal barriers (for example, owing to gastrointestinal perforation) promote invasive disease. A deeper understanding of specific Candida spp. virulence factors, host immune response and host susceptibility at the genetic level has led to key insights into the development of early intervention strategies and vaccine candidates. The early diagnosis of invasive candidiasis is challenging but key to the effective management, and the development of rapid molecular diagnostics could improve the ability to intervene rapidly and potentially reduce mortality. First-line drugs, including echinocandins and azoles, are effective, but the emergence of antifungal resistance, especially among Candida glabrata, is a matter of concern and underscores the need to administer antifungal medications in a judicious manner, avoiding overuse when possible. A newly described pathogen, Candida auris, is an emerging multidrug-resistant organism that poses a global threat.

Candidiasis is a broad term that refers to cutaneous, mucosal and deep-seated organ infections caused by fungi of the Candida genus, which can occur at any age and usually occur in the setting of easily identifiable risk factors for infection. Invasive candidiasis refers to bloodstream infections with Candida spp. (that is, candidaemia) and deep-seated infection - such as intra-abdominal abscess, peritonitis (inflammation of the peritoneum, the tissue that covers the inner wall of the abdomen and abdominal organs) or osteomyelitis (infection of the bones) - with or without candidaemia (FIG. 1).

Invasive candidiasis is an emerging infection closely linked to advances in medical technology and is widely recognized as a major cause of morbidity and mortality in the health-care environment ${ }^{1-6}$. At least 15 distinct Candida spp. can cause human disease, but the majority of invasive infections are caused by five pathogens: Candida albicans, Candida glabrata, Candida tropicalis, Candida parapsilosis and Candida krusei ${ }^{2-5}$. In certain parts of the world, a previously rare organism, Candida auris, has emerged as a major pathogen ${ }^{7-9}$ (BOX 1).

Candida spp. colonization is regarded as a pre*e-mail:ppappas@uabmc.edu is usually a consequence of increased or abnormal colonization together with a local or generalized defect in host defences. Invasive candidiasis is not a single clinical entity but rather is a disorder with myriad clinical manifestations that potentially affect any organ, as each Candida sp. possesses its own unique characteristics relative to invasive potential, virulence and antifungal susceptibility. Overall, C. albicans is the most common pathogen in most clinical settings, but non-albicans Candida spp. collectively could represent $>50 \%$ of the bloodstream isolates in certain regions ${ }^{2-5}$.

Key challenges to the management of candidaemia and invasive candidiasis include prevention, early recognition and rapid initiation of appropriate systemic antifungal therapy. Owing to the paucity of rapid diagnostic assays for invasive candidiasis, most clinicians rely on routine fungal cultures (which have low sensitivity) and empirical evidence (for example, unexplained fever or sepsis in a patient in the intensive care unit (ICU) with prior exposure to antibacterial agents, a central venous catheter and recent abdominal surgery) to establish a diagnosis. This approach can lead to the unnecessary use of antifungals in individuals without invasive candidiasis and also leads to delays in intervention with effective antifungal therapy for those who are infected. These delays in diagnosis and intervention could lead to much worse clinical outcomes. 


\section{Author addresses \\ ${ }^{1}$ Division of Infectious Diseases, University of Alabama at Birmingham, Birmingham, AL, USA. \\ ${ }^{2}$ Fungal Pathogenesis Section, Laboratory of Clinical \\ Immunology \& Microbiology, National Institutes of Allergy and Infectious Diseases, NIH, Bethesda, MD, USA. \\ ${ }^{3}$ Unit for Mycology, Statens Serum Institute, Copenhagen, Denmark. \\ ${ }^{4}$ Department of Clinical Microbiology, Rigshospitalet, Copenhagen, Denmark. \\ ${ }^{5}$ Department of Clinical Medicine, University of Copenhagen, Copenhagen, Denmark. \\ ${ }^{6}$ Division of Infectious Diseases, University of Texas Health \\ Science Center, Houston, TX, USA. \\ 'Department of Medicine and Radboud Center for \\ Infectious Diseases, Radboud University Medical Center, \\ Nijmegen, Netherlands.}

Antifungal resistance is an emerging problem worldwide, and this further complicates the selection of appropriate antifungal therapy. Candida spp. strains that are resistant to first-line antifungals (such as echinocandins and fluconazole) are increasingly being recognized, and their appearance usually correlates with high azole and/or echinocandin background usage in hospitals or specific hospital units ${ }^{10-14}$. The term multidrug-resistant (MDR) Candida sp. is used to designate Candida spp. strains that are resistant to two antifungal drug classes, whereas the term extensively drug-resistant (XDR) Candida sp. can be used to designate Candida spp. strains that are resistant to $\geq 3$ antifungal drug classes.

In this Primer, we discuss invasive candidiasis in adult patients. Candidiasis in neonates and other paediatric populations and cutaneous and mucosal Candida spp. infections (such as intertrigo (inflammation of body folds), oropharyngeal candidiasis and candidal vaginitis) are not reviewed here.

\section{Epidemiology}

The fungi

There is substantial geographical, centre-to-centre and unit-to-unit variability in the prevalence of Candida spp. (FIG. 2). C. albicans continues to be the most prevalent Candida sp. causing disease in both adult and paediatric populations, but the past decades have witnessed an increase in diagnoses of non-albicans candidaemia in both groups ${ }^{10,11}$. The prevalence of non-albicans Candida spp. in most regions is usually determined by factors such as background antifungal usage patterns in the region, individual patient risk factors and clonal outbreaks, that is, outbreaks involving a Candida spp. molecular strain that is unique in a health-care environment. In the United States and northwestern Europe, the second most common species is generally C. glabrata in the non-outbreak setting ${ }^{10,11}$. C. glabrata is also generally more-common among individuals of $>60$ years of age and among recipients of solid organ transplant ${ }^{2,6}$. In Latin America, Southern Europe, India and Pakistan, C. parapsilosis and/or C. tropicalis are much more frequently encountered than C. glabrata. C. krusei is the least common of the five major Candida spp., and it is most often found among patients with underlying haematological malignancies who have received antifungal prophylaxis with fluconazole $^{10,11}$. A very worrisome global trend is the emergence of C. auris ${ }^{7-9}$ (BOX 1).

\section{The disease}

In many developed countries, Candida spp. rank in the top three or four pathogens causing health-careassociated bloodstream infections, which also include Staphylococcus aureus, coagulase-negative staphylococci and Enterococcus spp. ${ }^{2,4}$. In the United States, Candida spp. have been reported to cause up to $22 \%$ of health-care-associated bloodstream infections based on data from the National Health and Safety Network; of note, this was a highly selected patient population comprising only patients receiving antimicrobials ${ }^{1}$. In a 2009 point prevalence survey of ICUs from around the world, Candida spp. accounted for $18 \%$ of infections reported ${ }^{15}$. Approximately $50 \%$ of episodes of candidaemia occur in the ICU setting, reflecting the complexity of illness usually associated with this infection. There are well-described risk factors associated with invasive candidiasis that apply to all hospitalized persons but especially to those in the ICU ${ }^{16,17}$. Some risk factors are intrinsic to the host or the disease state, whereas others are the result of iatrogenic interventions. The most common individual risk factors include the presence of an indwelling central venous catheter, exposure to broad-spectrum antibacterial agents, long-term ICU stay with or without assisted ventilation, recent major surgery, necrotizing pancreatitis, any type of dialysis, total parenteral nutrition and iatrogenic immunosuppression (BOX 2).

Disease burden. The incidence of candidaemia varies with geographical region, local epidemiology, age and other factors. Most large national surveys report an incidence of 3-5 per 100,000 persons in the general population and $1-2 \%$ of all medical and surgical ICU admissions ${ }^{2,6}$. Community-acquired candidaemia (that is, acquired in the community rather than in the hospital) is a somewhat new observation in the United States and other developed countries and reflects the increased utilization of long-term intravenous access devices (for example, peripherally inserted central catheters and tunnelled intravascular catheters) and parenteral outpatient antimicrobial therapy ${ }^{6,18}$. The attributable mortality among all patients with candidaemia has been reported to be between $10 \%$ and $47 \%$, but a moreaccurate estimate is probably $10-20 \%$, with the risk of death being closely related to increasing age, higher Acute Physiology and Chronic Health Evaluation II (APACHE II) scores, the infecting Candida sp. (for example, C. parapsilosis is less virulent than other Candida spp. and is generally associated with lower all-cause mortality), the use of immunosuppressive agents, pre-existing renal dysfunction and other comorbidities, venous catheter retention and specific antifungal treatment ${ }^{2,4,18}$. The attributable cost of candidaemia is reported to be $\sim$ US $\$ 40,000$ per patient ${ }^{18}$. 
a
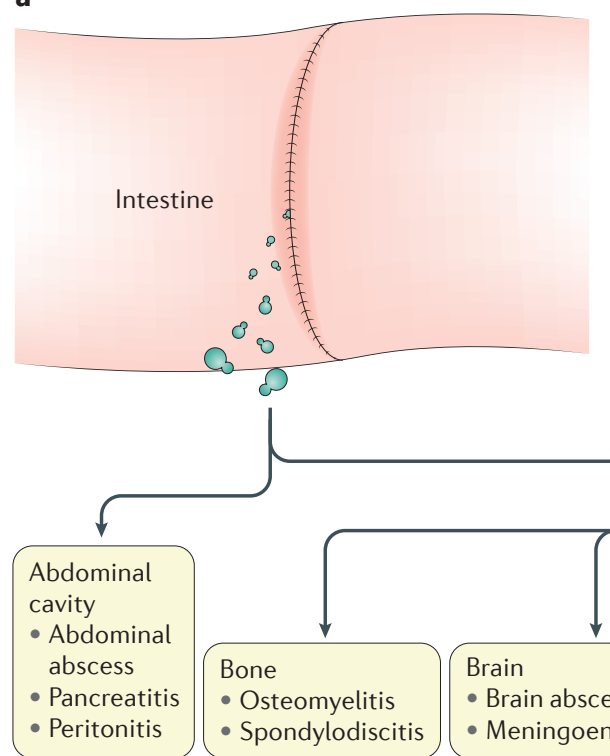

b

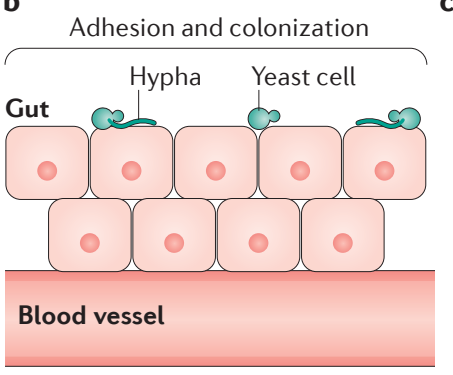

Hyphal penetration Vascular and invasion dissemination

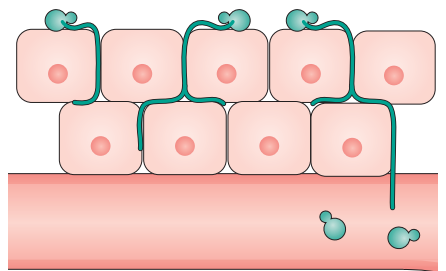

Cannula

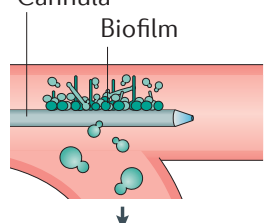

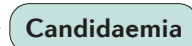
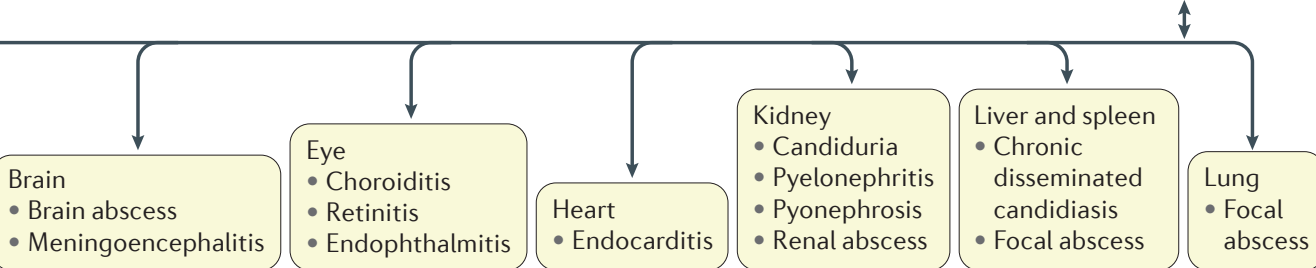

Figure 1 | Pathogenesis of invasive candidiasis. Candida spp. can be detected on the mucosal surfaces of $\sim 50-70 \%$ of healthy humans. a | When breaches in the intestinal barriers occur, for example, after gastrointestinal surgery, Candida spp. can disseminate to the abdominal cavity directly and invade the bloodstream (candidaemia). b | Under normal conditions, the fungus behaves as a commensal organism without causing disease. c l Impairment of immune response, among other factors, can promote fungal overgrowth in the gut and candidaemia, which can lead to deep-seated opportunistic infections in various organs (invasive candidiasis).

\section{Mechanisms/pathophysiology}

As invasive candidiasis is most often caused by C. albicans $^{2,4}$, this section will focus on this species, which has been the most extensively studied Candida sp. to date. However, the emergence of non-albicans Candida spp. has led to increasing studies of host-pathogen interactions in some of these species, predominantly C. glabrata and C. parapsilosis. Although common features of host immunity against these species and C. albicans have been reported ${ }^{19-20}$, additional studies are required to define the species-specific differences in cellular and molecular factors of protective immunity in these emerging Candida spp.

When perturbations of mucosal microbiota and/or weakening of host immunity occur, Candida spp. transition from commensalism to opportunism, which is associated with the induction of key virulence factors ${ }^{4,21-23}$. Specifically, there are three major conditions that predispose to human invasive infection. The first is long-term and/or repeated use of broad-spectrum antibiotics, which enable increased Candida spp. gut colonization. In fact, antibiotics confer Candida spp. a selective advantage over bacteria because commensal gut microbiota species are important in inducing the release of anti-Candida spp. protective factors from the mucosa; thus, depletion of these microbiota species by antibiotics removes these protective factors and enables Candida spp. overgrowth ${ }^{2,4,23}$. Another predisposing factor is breach of the gastrointestinal and cutaneous barriers by cytotoxic chemotherapy-induced mucositis (inflammation of the mucosa of the gastrointestinal canal), gastrointestinal surgery or perforation and/or central venous catheters, which collectively enable commensal
Candida spp. to translocate from mucocutaneous sites into the bloodstream (FIG. 1). The third factor is iatrogenic immunosuppression, such as chemotherapy-induced neutropenia or corticosteroid therapy, which impairs innate immune defences in tissues and thereby facilitates Candida spp. invasion from the bloodstream ${ }^{2,4}$ into organs such as the liver, spleen, kidneys, heart and brain. In contrast to mucosal candidiasis, in which T lymphocytes of the Thelper $17\left(\mathrm{~T}_{\mathrm{H}} 17\right)$ cell differentiation programme are crucial for host defence, effective immunity during invasive Candida spp. infection relies on myeloid phagocytes (neutrophils and mononuclear phagocytes, that is, monocytes, macrophages and dendritic cells (DCs)), not lymphocytes $22,24-26$.

\section{The fungus}

Several C. albicans virulence traits are integral to its ability to cause opportunistic infections and represent promising targets for vaccine and antifungal drug development. First, the ability of C. albicans to filament and interchange its morphotypes between unicellular yeast cells and pseudohyphae and hyphae (that is, multicellular, filamentous forms) critically promotes invasive disease, as strains locked in either the yeast or the filamentous form exhibit impaired virulence in mice ${ }^{27,28}$. Of note, this morphogenic transition is not required for virulence in C. glabrata, an important emerging cause of human invasive candidiasis despite its intrinsic inability to form hyphae ${ }^{2,4}$. The versatility of C. albicans to undergo morphogenic changes in response to various environmental factors also has important implications for immune evasion, because the differential composition and exposure of surface components (including 
mannoproteins (glycoprotein-containing mannose), glucans and chitin (a $\mathrm{N}$-acetylglucosamine polymer that is a major component of the cell wall)) in different Candida spp. morphotypes affect fungal sensing by the host innate immune system ${ }^{21,22,29-31}$. There are clinical strain-specific differences in fungal immune evasion and virulence ${ }^{30}$.

Second, C. albicans secretes a variety of factors in the context of invasive infection, including secreted aspartyl proteases and phospholipases that activate the innate immune response but are also important for promoting fungal tissue invasion and organ damage $e^{32,33}$. Candidalysin is a Candida spp. cytolytic peptide toxin ${ }^{34}$ that induces epithelial cell damage and activates danger signals during mucosal infection; however, its role in promoting the invasion of colonizing Candida spp. from the mucosa into the bloodstream and subsequent development of deep-seated infection is not well defined.

Third, effective adherence and invasion of Candida spp. in endothelial and epithelial cells enable their dissemination into the bloodstream ${ }^{35-37}$. Among the fungal proteins that mediate adherence, agglutinin-like protein 3 (encoded by als3), a C. albicans-specific adhesin and invasin, was targeted in the development of a fungal vaccine candidate in humans, which has now been successfully tested in a phase II clinical trial ${ }^{38}$. The capacity of Candida spp. for effective adherence also facilitates biofilm formation on implanted medical devices such as central venous catheters, which represents a major source of long-term candidaemia ${ }^{2,4,39}$ (FIG. 1). Much work has characterized the fungal regulatory circuits crucial for biofilm formation, which include transcriptional regulators and downstream target genes involved in adherence and filamentation ${ }^{39,40}$.

Drug resistance. Resistance in Candida spp. is either intrinsic (that is, found in all isolates within a species, such as fluconazole resistance in C. krusei) or acquired (that is, found in an isolate from a species that is normally susceptible, such as echinocandin resistance in C. glabrata). Echinocandin resistance in Candida spp. is emerging, particularly in C. glabrata. The target for the echinocandins is the $\beta$-D-glucan synthase enzyme, which is important for the cell wall synthesis. This enzyme is encoded by FKS1 in all Candida spp.

\section{Box 1 | Candida auris}

Candida auris, a pathogen originally described in Japan in 2009 that has reduced susceptibility to the major antifungal classes, is emerging as a cause of nosocomial outbreaks with rapid spread and high mortality ${ }^{7-9}$. C. auris was fluconazole resistant in $93 \%$ of patients; in $35 \%$ of patients C. auris was resistant to amphotericin B, in $7 \%$ was resistant to echinocandins, in $41 \%$ was multidrug resistant and in $4 \%$ was extensively drug resistant ${ }^{8}$. Outbreaks of $C$. auris have been reported in Asia (Japan, India and Pakistan), the United Kingdom, Spain, Colombia, Venezuela, Panama and the United States, and the list of countries continues to expand ${ }^{7-9}$. and is also encoded by FKS2 in C. glabrata. Mutations in two hotspot regions (HS1 and HS2) of these genes have been identified as the underlying mechanism for echinocandin resistance, where the level of resistance is dependent on the position of the mutated codon (and the corresponding amino acid), the specific amino acid alteration (which amino acid replaces the original one) and in which species the mutation occurs ${ }^{13,41,42}$.

Azoles target the enzyme encoded by ERG11. Acquired azole resistance is less common, and from a mechanistic point of view, it is often more complex, as several mechanisms including ERG11 mutations, upregulation of the target protein production and efflux pumps often play in concert ${ }^{43}$. Although multidrug resistance is a rare phenomenon ${ }^{44}$, mutations in $\mathrm{MSH} 2$, which encodes a DNA mismatch repair protein, in C. glabrata have been found in clinical isolates and have been demonstrated to facilitate selection of resistance to azoles, echinocandins and polyenes in vitro ${ }^{45}$. This mechanism may explain in part why echinocandin resistance in C. glabrata has been significantly linked to prior echinocandin and also fluconazole exposure or resistance, despite the fact that azoles and echinocandins have completely different targets in the cell (OR 3.6, 95\% CI 2.0-6.4) ${ }^{12,46}$.

\section{The host response}

Candida spp. recognition. The first step in developing anti-Candida spp. immune responses is recognition of extracellular or intracellular pathogen-associated molecular patterns (PAMPs) of invading Candida spp. yeast and hyphae by various families of soluble and membrane-bound pattern recognition receptors (PRRs) ${ }^{21,22,29}$. These PRRs are predominantly expressed by myeloid phagocytes and include Toll-like receptors (TLRs), C-type lectin receptors (CLRs), nucleotidebinding oligomerization domain (NOD)-like receptors, retinoid-inducible gene 1 protein (RIG1, encoded by RARRES3)-like receptors and complement components and receptors ${ }^{47-53}$ (FIG. 3). Candida spp. also activate the NLRP3 (NACHT, LRR and PYD domains-containing protein 3) and NLRP10 inflammasomes, which are multiprotein intracellular complexes that activate inflammatory responses. NLRP3 and NLRP10 activation results in the production of protective pro-inflammatory cytokines such as IL-1 $\beta$, IFN $\gamma$ and IL-17 (REFS 54-56). Besides Candida spp. sensing by individual PRRs, synergistic interactions between TLR2 and DC-associated C-type lectin 1 (dectin 1 , encoded by CLEC7A), galectin 3 or complement C5 anaphylatoxin (C5a), between TLR4 and C5a and between dectin 2 (encoded by CLEC6A) and dectin 1 or dectin 3 (encoded by CLEC4D) also occur, which broaden PAMP recognition and augment or curtail downstream responses ${ }^{22,29,48,57-60}$. Collectively, Candida spp. sensing by individual and/or synergistic PRRs initiates complex signalling cascades $^{47-49,61-64}$ that mediate the production of proinflammatory cytokines and chemokines; promote the recruitment, phagocytosis, generation of reactive oxygen species (ROS) and killing capacity of the phagocytes; and shape the activation of $\mathrm{T}_{\mathrm{H}}$ cell responses ${ }^{22,65,66}$. 


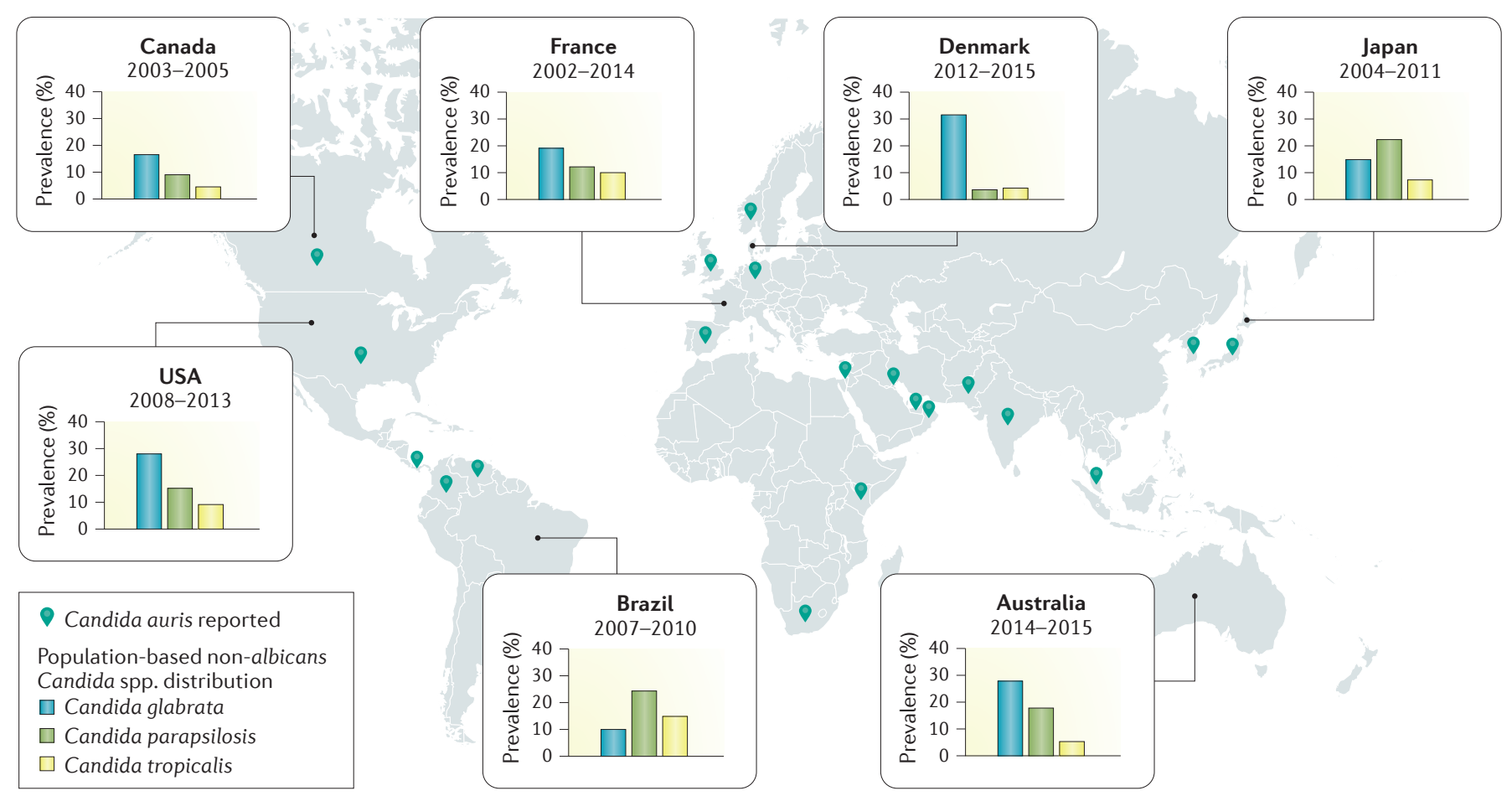

Figure 2 | Geographical variations in the distribution of Candida species. Globally, Candida albicans is the most prevalent species associated with invasive candidiasis; however, the distribution of non-albicans Candida spp. varies greatly, as exemplified in the representative countries shown. Of note, the species distribution may have changed since the data were collected. An increasing number of countries have reported cases of Candida auris infection. Data presented are from Australia, Brazil, Canada, Denmark, France, Japan and the United States ${ }^{6,200-205}$. Data on C. auris are from the Centers for Disease Control and Prevention (last accessed 27 March 2018).

Inborn errors of immunity (also known as primary immunodeficiencies) in various PRRs and downstream adaptor genes have shed light on the differential contributions of these pathways in host defence against human invasive candidiasis; undoubtedly, the most crucial PRR signalling cascade for human anti-Candida spp. immunity is that downstream of CLRs. Defects in the TLR and complement signalling pathways do not severely compromise the host's response to invading Candida spp. Indeed, patients with MYD88 (which encodes myeloid differentiation primary response protein MYD88) mutations, who lack TLR signalling and IL- $1 \beta$ receptor signalling, which is also dependent on MYD88, develop pyogenic bacterial infections but no invasive candidiasis ${ }^{67}$. Similarly, patients with genetic or acquired deficiencies in early complement components are primarily susceptible to invasive bacterial disease ${ }^{68}$. Conversely, patients with an autosomal recessive deficiency in the CLR pathway adaptor protein caspase recruitment domain-containing protein 9 (CARD9) have selectively heightened susceptibility to fungal infections (specifically, invasive candidiasis, chronic mucocutaneous candidiasis, extrapulmonary aspergillosis and phaeohyphomycosis) but not to bacterial or viral disease, which is consistent with the CLRCARD9 pathway being activated by Candida spp. and other fungal PAMPs ${ }^{65,66,69}$.

Strikingly, in individuals with CARD9 deficiency, invasive candidiasis typically affects the central nervous system (CNS) (although little is known on the mechanisms that enable Candida spp. to cross the bloodbrain barrier) but not the kidney, liver or spleen ${ }^{65,66,69,70}$. The aetiology for the CNS tropism of invasive candidiasis in CARD9 deficiency can be explained by the fact that resident glial cells and myeloid cells recruited to the Candida spp.-infected CNS require CARD9 to generate the chemoattractants that mediate neutrophil recruitment ${ }^{70}$. Of note, patients with null mutations in CLEC7A develop mucocutaneous but not invasive candidiasis, indicating that other CLRs upstream of CARD9 compensate for the lack of dectin 1 signalling in humans ${ }^{71}$.

Neutrophils. Neutrophils are essential for effective immunity during invasive candidiasis in mice, and neutropenia is a well-established risk factor for development of the infection and mortality in humans ${ }^{22,24,72,73}$. Neutrophil recruitment to the site of infection is of paramount importance for Candida spp. clearance, particularly early in the course of the infection, as in a mouse model, neutrophil accumulation is especially protective within the first $24-48$ hours after infection ${ }^{72}$. In mouse tissues, such as the liver and spleen, that recruit large numbers of neutrophils within the first 24 hours post-infection, Candida spp. growth can be successfully controlled, whereas the delayed trafficking of neutrophils into the Candida spp.-infected kidney is associated with ineffective renal host defence ${ }^{74}$. 


\section{Box 2 Risk factors for invasive candidiasis}

\section{General risk factors}

- Intrinsic: colonization with Candida spp., diabetes mellitus, gastrointestinal perforation, increased age, pancreatitis, sepsis and severity of illness.

- latrogenic: any type of dialysis (especially haemodialysis), broad-spectrum antibiotics, central venous catheter, corticosteroids and other immunosuppressants, gastrointestinal surgery or other major surgery, left ventricular assist device, long-term stay in hospital or intensive care unit, mechanical ventilation and total parenteral nutrition.

\section{Additional risk factors in patients with compromised immune system}

-Intrinsic: graft-versus-host disease, mucositis and profound neutropenia (absolute neutrophil count $<500$ cells $/ \mathrm{mm}^{3}$ ).

- latrogenic: solid organ transplant and stem cell transplant.
Assembly of the NADPH oxidase complex at the phagosomal membrane and activation of myeloperoxidase result in ROS generation, which promotes oxidative Candida spp. killing ${ }^{75-77}$ (FIG. 4). Dectin 1-dependent activation of both the calcineurin pathway and of the exchange factors for RHO-family GTPases protooncogene vav (encoded by Vav1) and guanine nucleotide exchange factor VAV3 (encoded by Vav3) also contributes to ROS formation in mouse neutrophils, indicating that patients treated with calcineurin inhibitors could have impaired anti-Candida spp. defences ${ }^{78,79}$. Indeed, NADPH oxidase-deficient and myeloperoxidasedeficient neutrophils from mice and humans exhibit impaired Candida spp. killing capacity, and a proportion of patients $(<5 \%)$ with chronic granulomatous disease or complete myeloperoxidase deficiency (two types of primary immunodeficiency) develop invasive candidiasis ${ }^{76,77,80}$. However, the majority of individuals with deficiency in the oxidative killing machinery never develop invasive candidiasis ${ }^{25,76,77}$, indicating that lack of ROS in human neutrophils can be largely compensated by non-oxidative killing mechanisms, whose importance is emerging ${ }^{47,81,82}$. Studies of patients with primary immunodeficiencies have shed light on the different phagolysosomal mechanisms that are employed by neutrophils for anti-Candida spp. killing when Candida spp. are opsonized compared with when the fungi are unopsonized $^{83,84}$ (FIG. 4).

Although neutrophils are crucial for host defence during invasive candidiasis, their functions that are aimed to control Candida spp. infection could come at the cost of immunopathology. In fact, excessive neutrophil accumulation in tissue late in the course of the infection is deleterious in mice, and certain pathways have been identified that may drive neutrophil-mediated immunopathology ${ }^{72}$. Indeed, pathogenetic neutrophil effects can be observed in patients with invasive candidiasis with renal involvement and in a subset of patients with neutropenia who develop invasive candidiasis with hepatosplenic involvement during the time of their neutrophil recovery ${ }^{85,86}$, and administration of corticosteroids has been employed in such situations to ameliorate the immune response-mediated tissue damage. Thus, pharmacological inhibition of these neutrophil-dependent immunopathology pathways may represent targeted therapeutic strategies in selected patients with invasive candidiasis.

Mononuclear phagocytes. In addition to neutrophils, other cell types including monocytes, macrophages and DCs are also important phagocytes for protection during invasive candidiasis ${ }^{87-89}$. Monocytes and tissue-resident macrophages exhibit marked efficiency in internalizing and ensnaring Candida spp. yeasts and filamentous forms in vivo within the first hours after infection and in producing pro-inflammatory cytokines and chemokines, which recruit and activate neutrophils in infected tissue ${ }^{87-89}$ (FIG. 4). Although less potent than neutrophils, monocytes and macrophages possess substantial anti-Candida spp. killing capacity ${ }^{87}$, which is accomplished at least in part via peroxynitrite production ${ }^{47}$.

Monocytes and macrophages also confer lymphocyteindependent protection following a subsequent occurrence of invasive candidiasis via epigenetic reprogramming, which results in 'innate memory' or 'trained immunity', that is, the ability of the innate immune system to mount resistance to reinfection ${ }^{90}$ (FIG. 3).

Moreover, $\mathrm{CD} 11 \mathrm{~b}^{+}$, but not $\mathrm{CD} 103^{+}$, DCs are indispensable for host defence during invasive candidiasis via the production of key inflammatory mediators, direct fungal killing - which is inferior to that of monocytes and macrophages - and priming of the candidacidal activity of neutrophils through an IL-23-granulocytemacrophage colony-stimulating factor (GM-CSF) pathway that involves natural killer cells ${ }^{91-95}$ (FIG. 4). Indeed, a randomized clinical trial demonstrated that recombinant GM-CSF therapy resulted in decreased mortality from invasive candidiasis in recipients of allogeneic haematopoietic stem cell transplant ${ }^{96}$.

\section{Immunogenetics for precision medicine}

The majority of patients in the ICU do not develop invasive candidiasis despite sharing similar risk factors, and the clinical outcome of the infection varies substantially among patients with similar clinical and microbiological risk factors. Thus, it is possible that individual genetic variations in key immune-related genes (singularly or in combination) confer heightened susceptibility to or protection from infection ${ }^{2,4,87,97-101}$. Indeed, population studies have demonstrated increases in susceptibility to invasive candidiasis in humans with selected genetic variants involving IL10, IL12B, TNF, CXCR1, CX3CR1, STAT1, PSMB8, SP110, CCL8, TLR1, CD58, TAGAP and LCE4A-C1orf68 (REFS 81,87,97-102).

In the first large prospective cohort of patients with candidaemia, in which patients in the ICU with the same underlying disease but without candidaemia were selected as matched controls, three TLR1 singlenucleotide polymorphisms (SNPs) were associated with susceptibility to candidaemia ${ }^{99}$. In another study, in patients in the ICU who developed candidaemia the presence of SNPs leading to increased circulating IL-10 and decreased IL-12b was associated with progression of disease and persistent candidaemia despite antifungal therapy ${ }^{97}$. The importance of the balance between 
pro-inflammatory and anti-inflammatory cytokines for the ability to clear the infection in humans was further underlined by a systems biology approach that identified the type I interferon pathway as the most prominent transcriptional response specifically activated by Candida spp. ${ }^{100}$. In the first genome-wide association study assessing genetic susceptibility to candidaemia, $\sim 119,000$ SNPs across 186 loci known to be associated with immune-mediated diseases were analysed in 217 patients with candidaemia, which is the largest cohort to date, and $\sim 12,000$ control individuals ${ }^{98}$. This approach identified three novel candidaemia genetic risk factors, mapping to CD58, the LCE4A-C1orf68 locus and TAGAP. Carrying two or more risk alleles (variants that are positively associated with candidaemia) of these loci increases the risk of candidaemia by 19 -fold ${ }^{98}$. Significant findings were confirmed in a clinical cohort of patients matched for disease. This 19-fold increased risk of developing candidaemia in individuals carrying specific SNPs suggests that it is feasible to develop screening strategies to identify patients in the ICU who are at risk and could benefit from antifungal prophylaxis. Further delineation of such genetic factors holds promise for devising immunogenetics-based risk stratification and prognostication strategies for identifying patients in whom targeted vaccination, antifungal prophylaxis and/or intensified diagnostic and therapeutic interventions could be beneficial.

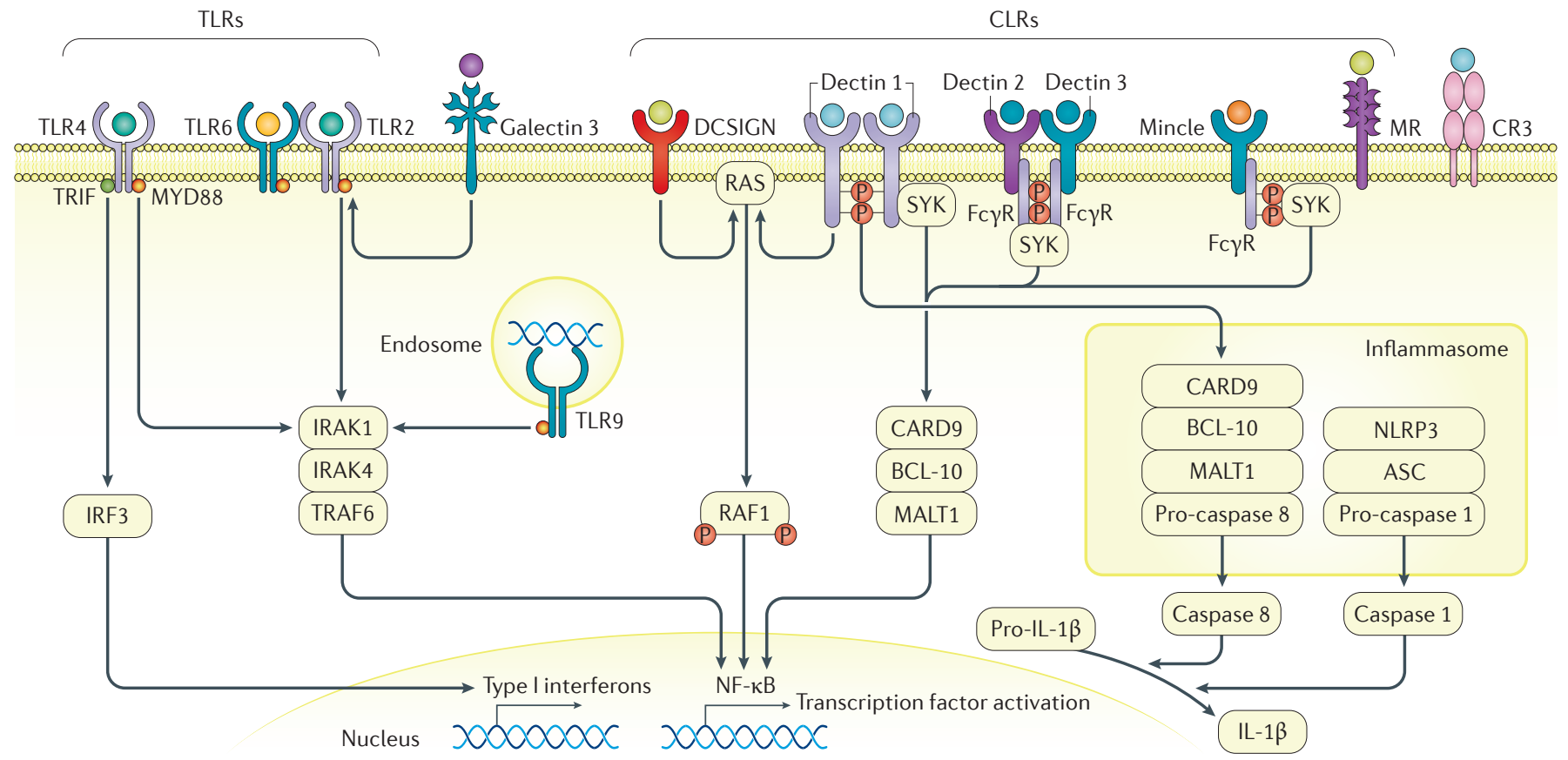

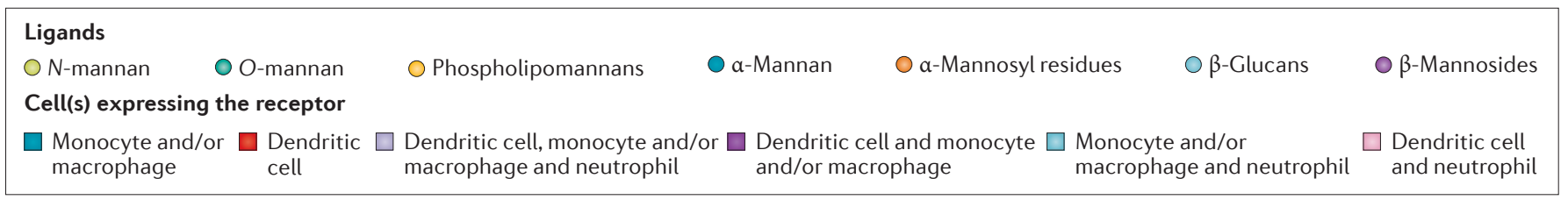

Figure 3 | Recognition of Candida spp. by pattern recognition receptors of myeloid phagocytes. Surface and intracellular pattern recognition receptors, the different myeloid phagocytes that express them and the corresponding Candida spp. pathogen-associated molecular patterns (ligands) that they recognize are shown. Cell-surface Toll-like receptor 4 (TLR4), intracellular TLR9 and cell-surface TLR2, which forms heterodimers with cell-surface TLR6, signal through the adaptor protein myeloid differentiation primary response protein MYD88. The C-type lectin receptor (CLR) family of receptors recognize fungal carbohydrates and result in activation of the spleen tyrosine kinase (SYK)-caspase recruitment domain-containing protein 9 (CARD9) axis, formation of the CARD9-B cell lymphoma/leukaemia 10 (BCL-10)-mucosa-associated lymphoid tissue lymphoma translocation protein 1 (MALT1) complex and nuclear factor- $\mathrm{kB}(\mathrm{NF}-\mathrm{\kappa} \mathrm{B}$ ) activation. Among CLRs, dendritic cell-associated C-type lectin 2 (dectin 2) forms heterodimers with dectin 3, and dectin 2, dectin 3 and macrophage-inducible C-type lectin (mincle, encoded by CLEC4E) require immunoglobulin- $\gamma$ Fc region receptor $(F c \gamma R)$ cooperation for signalling. The nucleotide-binding oligomerization domain (NOD)-like receptor NLRP3 (NACHT, LRR and PYD domains-containing protein 3) forms an inflammasome complex with apoptosis-associated speck-like protein containing a CARD (ASC) and caspase 1 that results in production of IL-1 $\beta$. In addition, dectin 1 signalling engages the CARD9-BCL-10-MALT1 complex to promote non-canonical inflammasome activation that results in IL-1 $\beta$ production via caspase 8 . Candida spp. $\beta$-glucans induce trained immunity in monocytes and macrophages via the dectin 1-RAF protooncogene serine/threonine-protein kinase (RAF1) signalling cascade through a pathway that involves RAC serine/threonine-protein kinase (AKT), hypoxia-inducible factor $1 \alpha(\mathrm{HIF} 1 \alpha)$ and serine/threonine-protein kinase mTOR and through a switch of cell glucose metabolism from oxidative phosphorylation to aerobic glycolysis (not depicted) ${ }^{206}$. CR3, complement receptor 3; DCSIGN, dendritic cell-specific ICAM3-grabbing non-integrin 1 (also known as CD209 antigen); IRAK, IL-1 receptorassociated kinase; IRF3, interferon regulatory factor 3; MR, mannose receptor; RAS, GTPase RAS; TRAF6, tumour necrosis factor receptorassociated factor 6; TRIF, TIR domain-containing adaptor molecule 1 (also known as TICAM1). Adapted from REF. 24. 


\section{Diagnosis, screening and prevention}

No clinical signs or symptoms are specific for invasive candidiasis. Thus, invasive candidiasis should be suspected in patients with known risk factors who have an unexplained fever that is unresponsive to antibacterial treatment. Timely diagnosis of invasive candidiasis is key to ensure a favourable outcome ${ }^{103,104}$. In fact, a 1-2-day delay in initiation of effective antifungal therapy has been associated with a doubling of mortality ${ }^{103,104}$. However, diagnosing invasive candidiasis can be challenging. Although the classical microbiological methods (light microscopy and culture) in general enable detection of Candida spp., concomitant presence of bacteria in clinical specimens can outcompete fungal growth. Thus, if invasive candidiasis is suspected, the diagnostic laboratory should be notified to use selective media (containing inhibitors of bacterial growth) and additional specific diagnostic options, such as antigen detection and PCR tests. Combinations of different diagnostic options could facilitate earlier and more-sensitive diagnosis (TABLE 1).

Distant sites of infection are quite uncommon among non-neutropenic patients with candidaemia. An estimated $15 \%$ of patients with candidaemia have

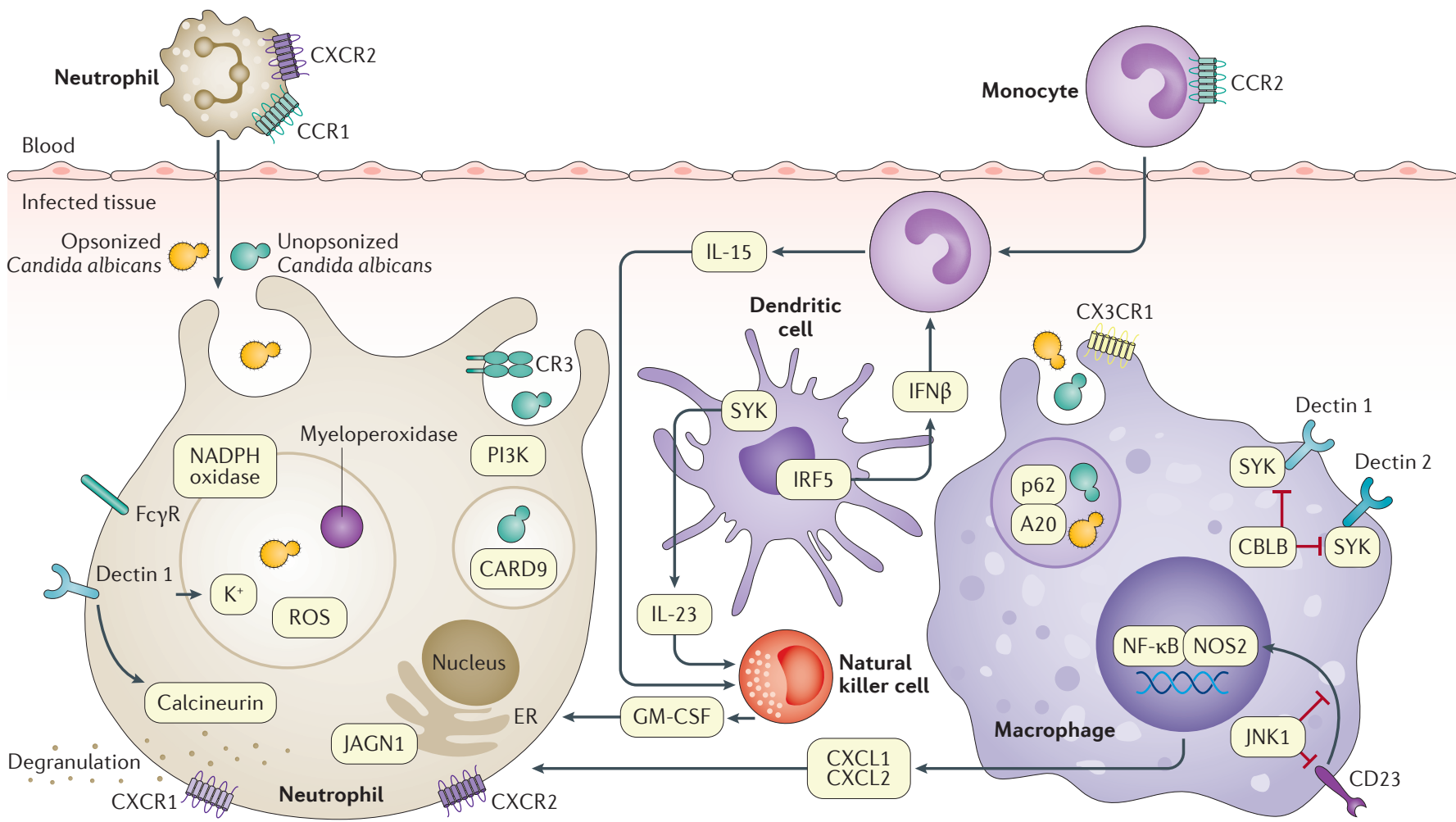

Figure 4 | Effector mechanisms of myeloid phagocytes for control of invading Candida spp. in infected tissue. Neutrophils, which are recruited into tissue via CXC-chemokine receptor 2 (CXCR2) (early phase) and CC-chemokine receptor 1 (CCR1) (late phase), are the myeloid cells with the most potent candidacidal activity and depend on both oxidative and non-oxidative mechanisms to kill Candida spp. The NADPH oxidase complex and dendritic cell-associated C-type lectin 1 (dectin 1)-calcineurin activation promote oxidative fungal killing. Differential signalling pathways are engaged during killing of opsonized (NADPH oxidase complex, immunoglobulin- $\gamma$ Fc region receptors (Fc $\gamma$ Rs) and protein kinase $\mathrm{C}$ ) and unopsonized (complement receptor 3 (CR3), caspase recruitment domain-containing protein 9 (CARD9) and phosphoinositide 3-kinase (PI3K)) fungal organisms. Neutrophil extracellular traps are released by neutrophils and contain antimicrobial proteins such as elastase and calprotectin, and they are thought to contribute to extracellular destruction of Candida spp. filamentous forms (not depicted). Extracellular damage to large Candida spp. filamentous forms is also mediated by neutrophil degranulation, which depends on jagunal homologue 1 (JAGN1) and the chemokine receptor CXCR1. Inflammatory monocytes are recruited to the Candida spp.-infected tissue in a CCR2-dependent manner, produce pro-inflammatory cytokines, kill Candida spp. and differentiate into inflammatory dendritic cells. CD11 b+ dendritic cells produce type I interferons (including IFN $\beta$ ) and also secrete IL-23 via spleen tyrosine kinase (SYK) activation; IL-23 acts on natural killer cells to produce granulocyte-macrophage colony-stimulating factor (GM-CSF), which potentiates the candidacidal activity of neutrophils in the Candida spp.-infected tissue. The IL-15 produced by CCR2-expressing inflammatory monocytes also stimulates natural killer cells to release GM-CSF for neutrophil activation. Tissue-resident macrophages effectively internalize Candida spp. yeast forms, encircle Candida spp. filamentous forms, exert candidacidal activity and produce a variety of pro-inflammatory cytokines and CXC-chemokines via the autophagic adaptor ubiquitinbinding protein p62 (also known as sequestosome 1), which sequesters zinc-finger protein A20 (also known as tumour necrosis factor $\alpha$-induced protein 3 ) in the autophagosome. Resident macrophage survival, accumulation in tissue and contact with Candida spp. in vivo depend on the $\mathrm{CX}_{3} \mathrm{C}$-chemokine ligand 1 (CX $\left.{ }_{3} \mathrm{CL} 1\right)-\mathrm{CX}_{3} \mathrm{C}$-chemokine receptor 1 (CX3CR1) axis in both mice and humans ${ }^{87,207-209}$. Inhibition of SYK-dependent dectin 1 and dectin 2 signalling in macrophages by the E3 ubiquitin-protein ligase CBLB impairs macrophage effector functions. Similarly, inhibition of the C-type lectin receptor CD23 signalling by JUN N-terminal kinase 1 (JNK1; also known as mitogen-activated protein kinase 8) results in impaired macrophage production of nitric oxide and defective fungal killing. Thus, CBLB and JNK1 could represent potential therapeutic targets ${ }^{207,210}$. CXCL, CXC-chemokine ligand; ER, endoplasmic reticulum; IRF5, interferon regulatory factor 5; NF-kB, nuclear factor-kB; NOS2, nitric oxide synthase, inducible; ROS, reactive oxygen species. 
Table 1 | Specimen types and tests for the diagnosis of invasive candidiasis

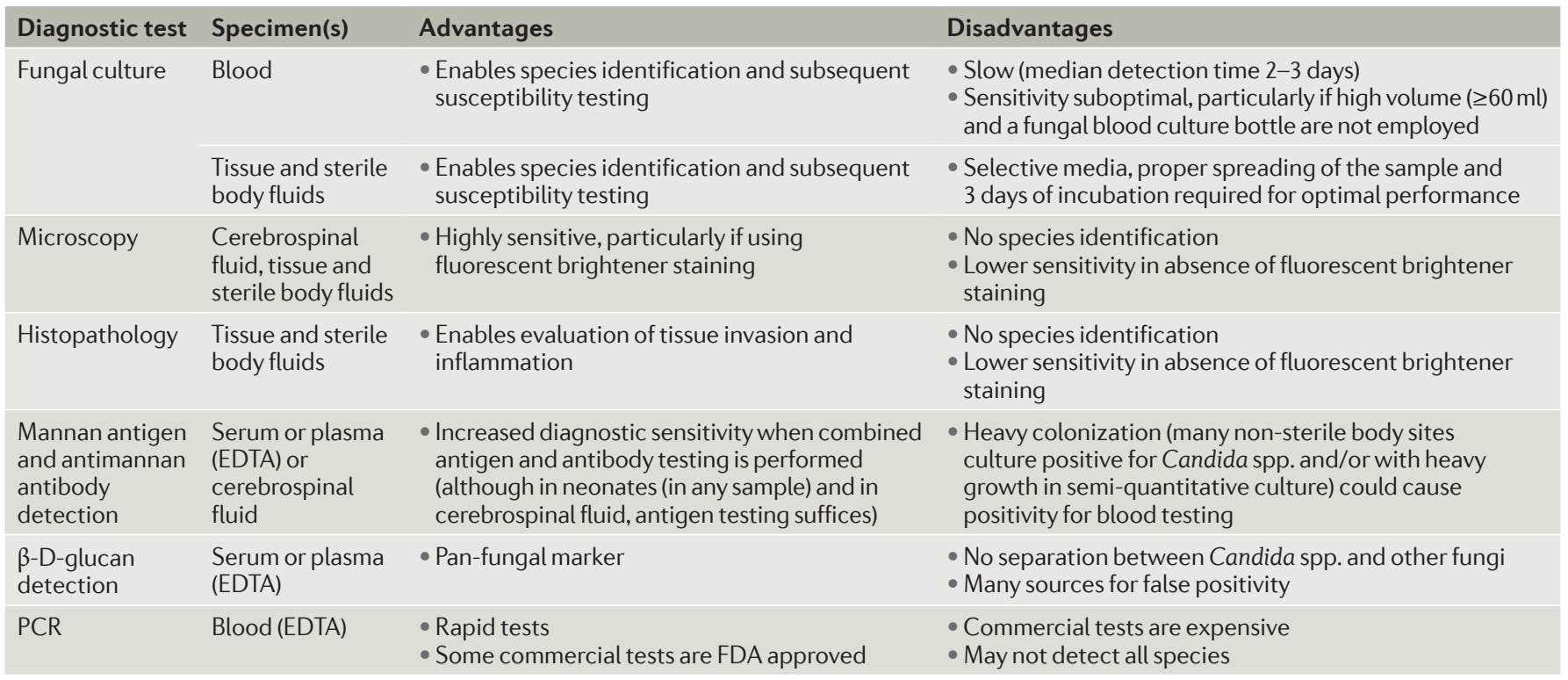

some manifestation of ocular involvement, and 1-2\% will develop severe, sight-threatening endophthalmitis (inflammation of the retina, choroid and vitreous humour $)^{105}$. For all patients with candidaemia, a dilated funduscopic examination performed by an experienced clinician is indicated within the first week after initiation of specific antifungal therapy ${ }^{106,107}$. Among patients with candidaemia in the setting of neutropenia, the funduscopic examination should be delayed until neutrophil recovery because the characteristic inflammatory retinal and choroidal changes might not become clinically evident during periods of profound neutropenia ${ }^{106}$. Endocarditis is an uncommon complication of candidaemia, and the routine performance of an echocardiography among patients with candidaemia is not generally recommended unless there are risk factors present for endocarditis such as intravenous drug abuse, pre-existing valvular disease or the presence of a prosthetic cardiac valve.

\section{Classical diagnostic techniques}

Blood culture remains the gold standard for diagnosis of invasive candidiasis, but it is an insensitive tool, as it is positive in $21-71 \%$ of patients with autopsy-proven invasive candidiasis depending on sampling frequency and volume of blood drawn ${ }^{108}$. Nonetheless, it is an essential diagnostic test and enables species identification and susceptibility testing ${ }^{106,108-110}$. During an episode of candidaemia, the number of circulating Candida spp. cells is usually $<1$ colony forming unit (CFU) per $\mathrm{ml}$ (REF. 111). Candida spp. multiply more slowly than bacteria, a fact that partly explains the suboptimal sensitivity and prolonged time to positivity (median of 2 days) for blood cultures ${ }^{111,112}$. Frequent sampling (once daily or more frequently if the patient has a fever), larger blood culture volumes (optimally $\geq 40-60 \mathrm{ml}$ daily), the use of specialized fungal blood culture bottles with selective medium and sampling before initiation of antifungal therapy increase sensitivity ${ }^{110,112-116}$. For noncandidaemic invasive candidiasis, diagnosis is firmly established based on positive culture for Candida spp. and/or positive histopathology from normally sterile sites, such as intra-abdominal and intra-thoracic sites. Tissue and/or cytology specimens from normally sterile sites or newly placed drains (placed within the past $24 \mathrm{~h}$ ) should undergo microscopy, preferably using staining with a fluorescent brightener, and also be sent for histopathological examination using special staining for fungi (Grocott-Gomori methenamine silver stain, which recognizes carbohydrates in the fungal cell wall, and periodic acid-Schiff, which detects polysaccharides and glycoproteins).

As early as in the mid-1980s, a matched case-control study showed that isolation of Candida spp. from sites other than blood was associated with candidaemia (OR 10.37) ${ }^{117}$. Subsequent genotyping studies have confirmed the strong association between the colonizing and invasive Candida sp. isolates obtained from the same individual, supporting the concept that invasive infection usually arises from the colonizing microbiota organisms owing to a barrier breech ${ }^{118,119}$. Consequently, cultures from non-sterile body sites yielding the presence of colonizing Candida spp. can be informative regarding likelihood of invasive candidiasis ${ }^{120}$ and regarding which species is most likely involved; such information can be used to select antifungal therapy in patients in whom the diagnosis is suspected but not confirmed by culture from a normally sterile site. In an attempt to optimize the predictive value of such surveillance cultures from non-sterile sites, studies were undertaken to determine whether the number of body sites and degree of colonization (light, moderate or heavy Candida spp. growth) at these body sites were crucial to the development of candidaemia. A colonization index $\geq 0.5$ (the ratio of the number of Candida spp. positive specimens to the number of samples taken) and a corrected colonization 
index $\geq 0.4$ (the colonization index multiplied by the proportion of sites with heavy growth (quantified as +++ from semi-quantitative culture) to the total number of body sites with Candida spp.) were developed and associated with invasive candidiasis in some $e^{16,121}$ but not all studies $^{122}$. However, regular routine surveillance cultures for patients in the ICU who are at risk of invasive candidiasis are labour intensive and not broadly used. A 2015 study examining cultures obtained 72 hours after ICU admission and then twice weekly showed that culture of throat and perineum samples within the first two time points after ICU admission captures $84 \%$ (61/73 patients) of patients with subsequent invasive candidiasis, with negative predictive values of $99 \%{ }^{120}$, but a randomized empirical treatment study failed to show a correlation between empirical antifungal therapy based on positive colonization cultures at study inclusion (from mouth, throat, upper respiratory tract, skin, urine and digestive tract (rectal swab or faeces) and, if appropriate, drains and catheters) and patient survival in non-neutropenic patients with sepsis and organ failure in the ICU ${ }^{122}$.

\section{Antigen detection}

Mannan antigen and antimannan antibody. Candida spp. mannan antigen and antimannan antibody detection can be useful for the detection of invasive candidiasis ${ }^{123-127}$, including in paediatric patients and in those with CNS infection ${ }^{123,128-130}$. However, in a 2016 study including patients in the ICU with severe abdominal conditions, the performance of combined mannan antigen and antimannan antibody detection was disappointing (sensitivity $55 \%$ and specificity $60 \%$ ) $^{131}$. Antibodies are often present in patients with compromised immune system with prior candidaemia or heavy colonization; thus, the positive predictive value of detection of antibodies alone in the absence of rising concentration is ${ } \mathrm{w}^{123,126}$. This observation and the unexplained variable performance across various studies are important caveats that could lead to inappropriate antifungal treatment of patients who are unlikely to have candidiasis unless the clinicians understand the strengths and limitations of the tests. Candida spp. mannan and antimannan antibody detection is not US FDA approved as a diagnostic tool in the United States and is mainly available in Europe.

$\beta$-D-glucan. $\beta$-D-glucan is a pan-fungal marker of invasive fungal infection. The antigen can be detected in blood during Candida spp., Aspergillus spp., other mould (not Mucorales) and Pneumocystis jirovecii infections ${ }^{132,133}$. Hence, the test cannot distinguish between candidiasis and infections caused by the other fungi. The sensitivity for invasive candidiasis was generally high (76.7-100.0\%) in seven studies from 2011 to 2018 , with half of the studies reporting sensitivity above $85.0 \%$. However, the specificity was more-variable $(40.0-91.8 \%)^{131,134-139}$. Multiple sources for false positivity have been reported, including heavy Candida spp. colonization without infection ${ }^{131}$, haemodialysis with cellulose membranes, human blood products (immunoglobulins or albumin), antibiotics (amoxicillin-clavulanate or piperacillin-tazobactam), serious bacterial infections, surgical sponges and gauzes containing glucan and severe mucositis, which is particularly challenging in the ICU and haematological setting, and these sources often lead to lower specificities ${ }^{131,134,140}$. However, many of these sources are often present in the same patient, making it difficult to dissect which are more-important and whether the risk of contamination is limited to certain types or batches of each of these sources. Nevertheless, the specificity was below $60 \%$ in half of the studies, illustrating that false positivity is a recurrent challenge. The negative predictive value has been high in most studies, reflecting a high sensitivity and suggesting that invasive candidiasis, including candidaemia, is unlikely when the $\beta$-D-glucan test result is negative.

C. albicans germ tube antigen. Another Candida spp.-specific antibody test is the C. albicans germ tube antigen (CAGTA) test; the antigen was originally found in C. albicans, but the test also detects candidiasis with other Candida spp., although it detects some nonalbicans Candida spp., including C. parapsilosis, with lower sensitivity ${ }^{131,138,141,142}$. The experience with this test is more limited than that with Candida spp. mannan antigen and antimannan antibody and $\beta$-D-glucan tests. The sensitivity ranges from $53.3 \%$ to $74.1 \%$, and specificity ranges from $56.5 \%$ to $92.0 \%{ }^{131,138,141,142}$.

Combination tests. As none of the above-mentioned antigen and antibody tests are perfect, several studies have investigated the performance of various combinations of these tests or of the combination of one of these and DNA-based techniques. For example, positive CAGTA antibody and $\beta$-D-glucan tests in a single blood sample or $\beta$-D-glucan positivity in two consecutive blood samples enabled the discrimination of invasive candidiasis from Candida spp. colonization in critically ill patients with severe abdominal conditions ${ }^{131}$. The combination of CAGTA and $\beta$-D-glucan or CAGTA and Candida spp. mannan antigen had a very high negative predictive value and could be used for discontinuing unnecessary empirical therapy in patients with suspected candidaemia ${ }^{138}$. The drawback of such approaches is increased cost, and if two consecutive positive tests are required, the diagnosis can be delayed, unless the tests are offered on a daily basis. As procalcitonin testing is available on a daily basis in most institutions, the combination of $\beta$-D-glucan and procalcitonin testing has been evaluated: the combination of a positive $\beta$-D-glucan test with low procalcitonin $(<2 \mathrm{ng} / \mathrm{ml})$ had a sensitivity and specificity of $66 \%$ and $98 \%$ for invasive candidiasis, respectively, whereas if only one of these two criteria was met, the performance parameters were $96 \%$ and $60 \%{ }^{139}$.

\section{DNA-based techniques}

The development and implementation of PCR-based tests for the detection of fungal infections in daily routine lag behind those for viral and bacterial infections. The main challenges have been efficacious DNA extraction from the fungal cells, low numbers of fungal cells in blood, similarity between fungal and human DNA and 
the ubiquity of many fungal pathogens or their DNA in the environment, on skin, in buffers and on utensils leading to false positive results. Thus, the performances reported for locally developed PCR assays vary substantially, and PCR-based tests have not yet been incorporated into official guidelines or classification criteria for Candida spp. disease ${ }^{106,143}$.

Candida spp. can be detected by various commercially available PCR assays targeting bacterial and fungal pathogens; these assays can be used in patients with sepsis and include the LightCycler SeptiFast Test MGRADE (Roche Molecular Diagnostics, Pleasanton, CA, USA), SepsiTest (Molzym Molecular Diagnostics, Bremen, Germany), the IRIDICA BAC BSI assay (Abbott Diagnostics, Lake Forest, IL, USA), which uses PCR and electrospray ionization mass spectrometry technology, and a test targeting Candida spp. and Aspergillus spp. using the RenDx Fungiplex assay (Renishaw Diagnostics, Glasgow, United Kingdom $)^{144-148}$. SeptiFast is the most thoroughly evaluated system. Although the numbers of patients with candidaemia are low in all studies, SeptiFast detected more Candida spp.-positive specimens than blood culture, especially from patients receiving antifungal therapy, and produced a Candida spp. distribution mirroring that found in blood cultures ${ }^{149-152}$. Drawbacks are that the test is labour intensive, that only the five most common Candida spp. are included and that the sensitivity for detecting the haploid species C. glabrata is lower $(100 \mathrm{CFU} / \mathrm{ml}$ are required for $100 \%$ sensitivity). Nonetheless, these studies support that DNA-based assays for Candida spp. can potentially improve timeliness and sensitivity of the diagnosis. In contrast to these broad-spectrum sepsis tests, the T2Candida Panel test (T2 Biosystems, Lexington, MA, USA) is an FDAapproved test for diagnosis of candidaemia specifically. It detects the five most common Candida spp. from whole blood and additionally detects Saccharomyces cerevisiae, Candida bracarensis, Candida metapsilosis and Candida orthopsilosis. However, the test cannot identify a specific species, and the results fall into one of the three following groups: C. albicans or C. tropicalis; C. glabrata, C. krusei, S. cerevisiae or C. bracarensis; or C. parapsilosis, C. metapsilosis or C. orthopsilosis. The pooled sensitivity and specificity were $91.1 \%$ and $99.4 \%$, respectively, in a study combining data from approximately 1,500 clinical samples and 300 artificial positive control samples created with various concentrations of the five most common species ${ }^{153}$. In practice, the sensitivity might be somewhat lower depending on the prevalence of candidaemia caused by species not covered by the T2Candida Panel test. Such species are responsible for $\sim 5 \%$ of candidaemia infections according to a nationwide fungemia programme in Denmark but can exceed $20 \%$ in selected settings ${ }^{154-156}$; of note, the test does not detect the emerging MDR species C. auris. The test seems promising and cost effective in recent studies ${ }^{156,157}$.

\section{Drug susceptibility testing}

Once the diagnosis of invasive candidiasis is confirmed, it is important to establish the susceptibility profile of the identified species. Intrinsic resistance is diagnosed by a correct species identification, whereas identification of acquired resistance of the specific isolate requires correct susceptibility testing and application of clinical breakpoints. Clinical breakpoints are used for categorizing the susceptibility test result as susceptible, intermediate or resistant and for everyday use in the clinical laboratory to advise on patient therapy.

The Clinical and Laboratory Standards Institute (CLSI) and the European Committee on Antimicrobial Susceptibility Testing (EUCAST) have developed reference standards for antifungal susceptibility testing with associated clinical breakpoints for most common species-compound combinations ${ }^{158-161}$. Both tests are microbroth dilution tests, where the ability of the Candida sp. isolate to grow in serial dilutions of the antifungal agent is investigated. The lowest concentration that causes prominent or at least $50 \%$ growth inhibition compared with an uninhibited control is the test result, the MIC (minimum inhibitory concentration). These are excellent methodologies for identifying resistant isolates, but they are not user friendly in the context of the routine clinical microbiology laboratory. A range of other commercial susceptibility tests is available, and none is superior across all organism-antifungal combinations, emphasizing that local validation and experience are important ${ }^{162-165}$ (TABLE 2). The most common are agar diffusion tests (disk and gradient strips), commercial microbroth dilution tests or the semi-automated system VITEK (bioMérieux, Marcy l'Etoile, France).

\section{Prevention}

Antifungal prophylaxis and pre-emptive therapy. Most published data suggest that antifungal prophylaxis with fluconazole reduces the incidence of invasive candidiasis in patients in the ICU who are at high risk of developing the infection, but reduction of mortality has not been convincingly reported ${ }^{166,167}$. Of note, antifungal prophylaxis could theoretically favour the emergence of acquired resistance. Data supporting the use of targeted prophylaxis among patients with certain risk factors of invasive candidiasis are conceptually promising, but the results of clinical trials have been unconvincing $^{168}$. Stronger data support the use of targeted antifungal prophylaxis in selected high-risk recipients of liver, pancreas, small bowel or haematopoietic stem cell transplant ${ }^{106}$. Much less clear is the value of pre-emptive therapy (that is, based on positive biomarkers, such as $\beta$-D-glucan, during patient monitoring at frequent intervals before blood or tissue culture yield positive results) and of empiric antifungal therapy among patients at high risk of invasive candidiasis with unexplained symptoms but without clear mycological evidence of disease. Several studies have attempted to define a clear role for antifungals in this setting but have failed to do so convincingly ${ }^{121,122,169-171}$.

Infection prevention and hospital epidemiology. Practical measures to prevent infection with Candida spp. include judicious use of antibacterials and mitigation of some of the preventable risk factors mentioned previously ${ }^{16-18,172-175}$. Candida spp. (and other yeasts) can 
Table 2 | Overview of various available antifungal susceptibility tests

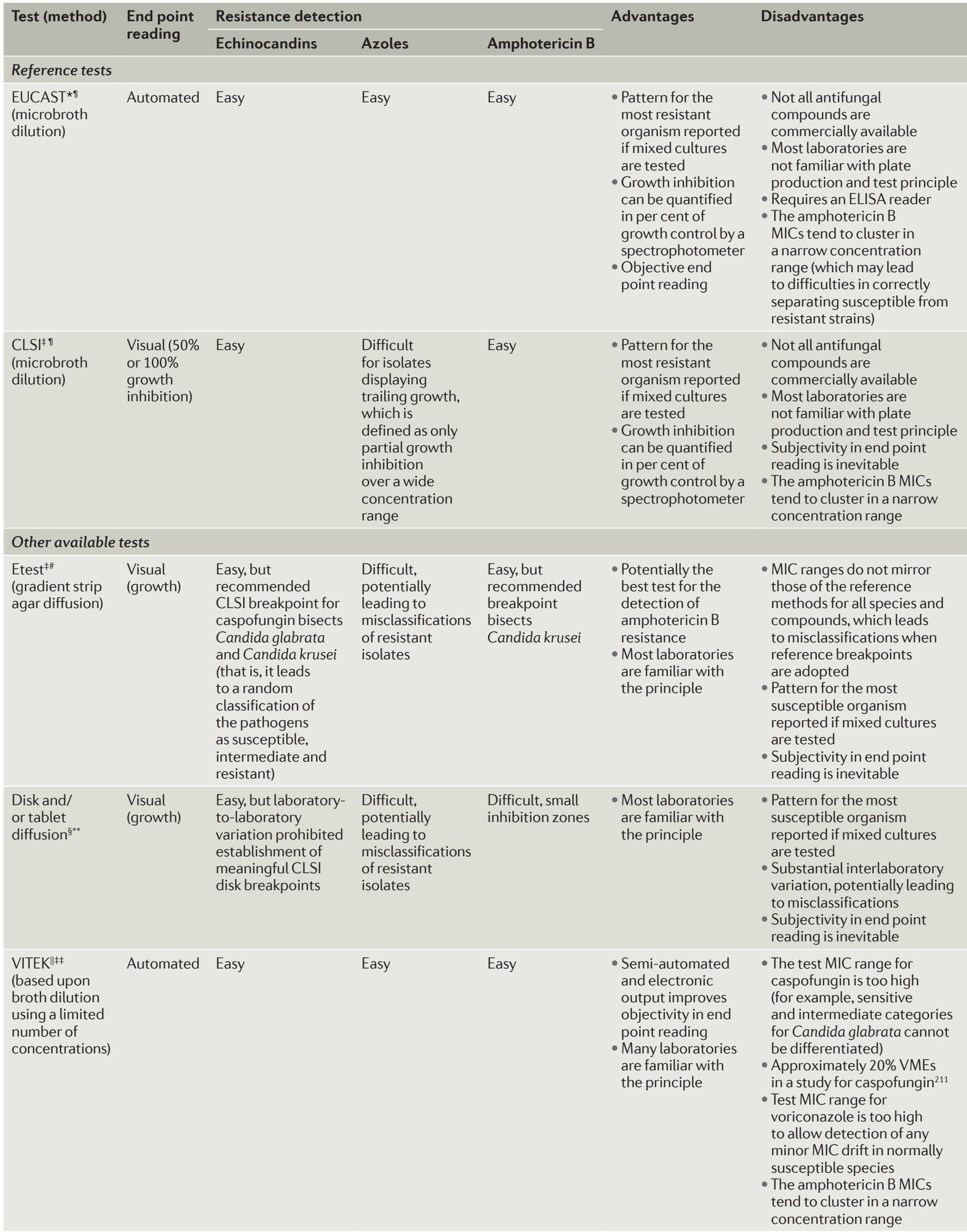


Table 2 (cont.) | Overview of various available antifungal susceptibility tests

\begin{tabular}{|c|c|c|c|c|c|c|}
\hline \multirow[t]{2}{*}{ Test (method) } & \multirow{2}{*}{$\begin{array}{l}\text { End point } \\
\text { reading }\end{array}$} & \multicolumn{3}{|c|}{ Resistance detection } & \multirow[t]{2}{*}{ Advantages } & \multirow[t]{2}{*}{ Disadvantages } \\
\hline & & Echinocandins & Azoles & Amphotericin B & & \\
\hline \multicolumn{7}{|c|}{ Other available tests (cont.) } \\
\hline
\end{tabular}

CLSI, Clinical and Laboratory Standards Institute; ECV, epidemiological cut off value (that is, the MIC value that describes the upper MIC value for isolates without acquired resistance mechanisms); ELISA, enzyme-linked immunosorbent assay; EUCAST, European Committee on Antimicrobial Susceptibility Testing; MIC, minimum inhibitory concentration; VME, very major error. * Low sensitivity to inoculum variation. ${ }^{\ddagger}$ Medium sensitivity to inoculum variation. ${ }^{\text {. } H i g h ~ s e n s i t i v i t y ~ t o ~}$

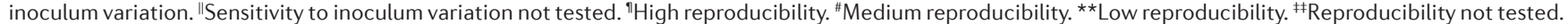

also spread through nosocomial transmission: they can be transferred to patients via hands of health-care workers or contaminated material (for example, intravenous saline for central venous catheter flushing shared among patients), which can cause outbreaks ${ }^{176-180}$. C. parapsilosis is most commonly associated with nosocomial transmission, targeting neonates, patients with immunosuppression and patients with indwelling catheters. Some observations suggest person-to-person transmission between hospitalized patients may occur more frequently than previously estimated. Data from molecular typing studies suggest that minor clusters of infections involving isogenic isolates occur regularly ${ }^{181,182}$. Moreover, data from the Atlanta-Baltimore Centers for Disease Control and Prevention (CDC) surveillance programme describe an exceptionally high proportion of echinocandin-resistant C. glabrata with FKS mutations among echinocandin naive patients, suggesting nosocomial transmission ${ }^{12}$. Thus, strict adherence to routine infection prevention procedures including hand hygiene and central venous catheter management are crucially important. In this regard, the adoption of 'catheter care bundles' (a standard combination of interventions to prevent catheter-associated bloodstream infections) as an approach to central venous catheter management has been important in reducing catheter-associated bloodstream infections.

\section{Management}

In addition to early diagnosis, two clinical interventions are essential to the successful management of invasive candidiasis: source control and early initiation of treatment with early effective systemic antifungal therapy, usually before the diagnosis of invasive candidiasis has been confirmed. Source control refers to the elimination of the suspected nidus (focus) of infection, such as removal of contaminated intravascular catheters and effective drainage of collections of infected material, for example, peritoneal fluid, pleural fluid and/or abscess material. Removal of these presumably infected prosthetic devices, such as cardiac pacemaker leads, prosthetic joints or other devices, when feasible, is important to a successful outcome. In addition to source control, early effective antifungal therapy is crucial in the successful treatment of patients with invasive candidiasis, as data indicate significantly higher mortality in patients with candidaemia when antifungal therapy was delayed or inadequate (for example, the drugs were not chosen on the basis of susceptibility data or the dosage was insufficient) and/or when source control was not quickly achieved ${ }^{183-185}$. There are several published guidelines outlining expert recommendations for the management of invasive candidiasis and candidaemia, with detailed recommendations for specific clinical circumstances ${ }^{106,107}$.

\section{Initial therapy}

The selection of an antifungal drug for initial treatment should be based on the patient's prior exposure or intolerance to an antifungal agent, severity of illness, relevant comorbidities and involvement of the brain, cardiac valves and/or visceral organs. The working knowledge of the main Candida spp. and susceptibility data in a particular clinical unit should also be considered.

Echinocandins. Most clinicians choose an echinocandin (anidulafungin, caspofungin or micafungin) as first-line therapy for adult patients (FIG. 5). Echinocandins inhibit $\beta$-D-glucan synthase, an enzyme required for the formation of the cell wall, and have excellent fungicidal activity against most Candida spp. Echinocandins are effective, safe and have very limited drug-drug interactions; however, they require intravenous administration. Each agent in this class was effective in $\sim 70-75 \%$ of patients in randomized, comparative clinical trials ${ }^{186-190}$.

An analysis of seven large-scale randomized clinical trials that compared treatment for invasive candidiasis in almost 2,000 patients overall showed that an echinocandin as the first-line agent for initial therapy was associated with significantly lower 30-day mortality 


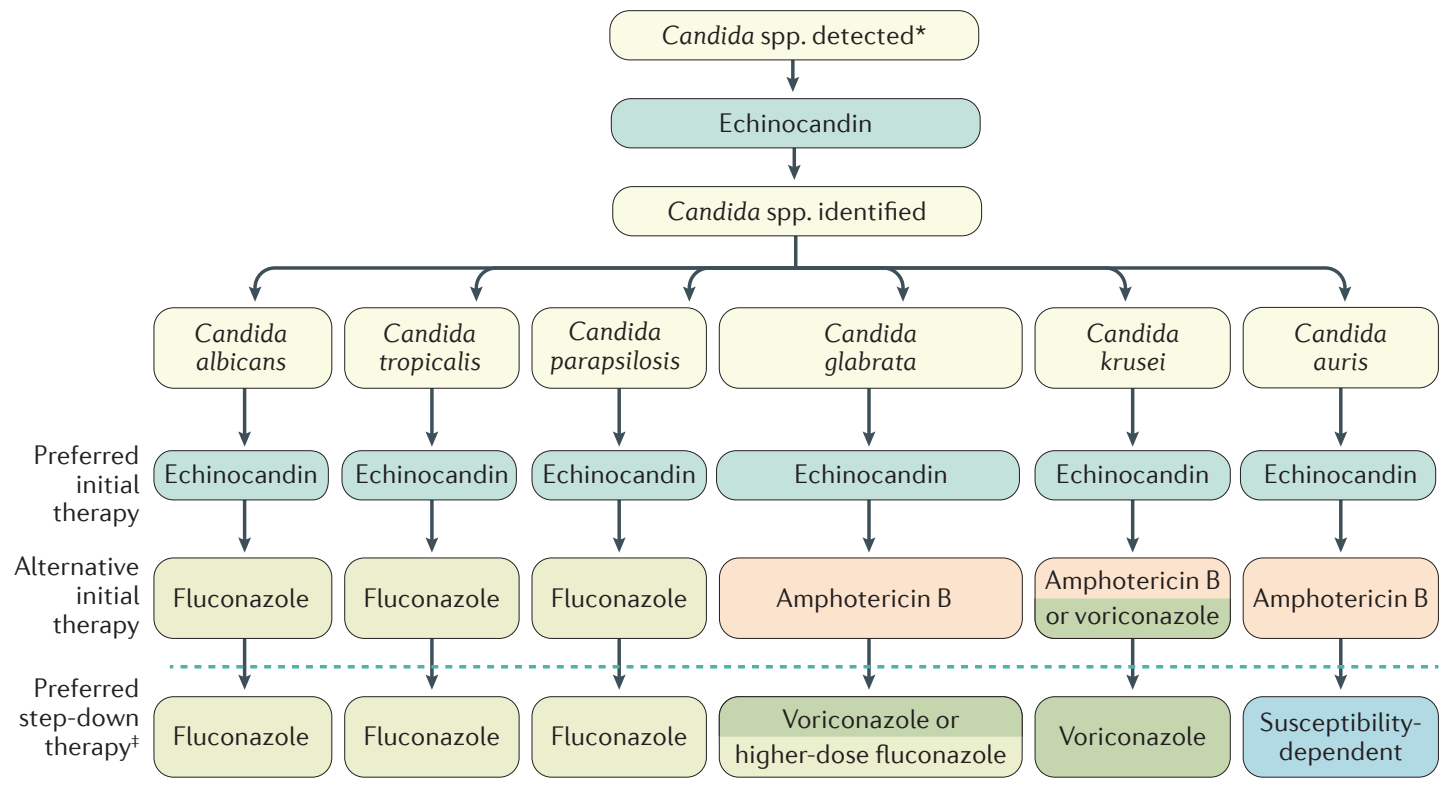

Figure 5 | Algorithm for the management of invasive candidiasis. For candidaemia, the total duration of therapy is 14 days from the first negative blood culture. *As yet unknown species. ${ }^{*}$ Step-down therapy to fluconazole is usually based on documented susceptible minimum inhibitory concentrations to fluconazole $(<2 \mu \mathrm{g} / \mathrm{ml}$ for C. albicans, C. parapsilosis and C. tropicalis and $<32 \mu \mathrm{g} / \mathrm{ml}$ for C. glabrata) and clinical stabilization of the patient. Higher-dose fluconazole consists of $12 \mathrm{mg} / \mathrm{kg}$ per day. The information in the presented figure is based in part on Infectious Diseases Society of America (IDSA) and European Society of Clinical Microbiology and Infectious Diseases (ESCMID) guidelines ${ }^{106,107}$.

compared with an azole or amphotericin $\mathrm{B}(\mathrm{AmB})^{185}$. Higher APACHE II score, older age, intravascular catheter retention and infection with C. tropicalis were associated with worse outcomes and higher mortality, irrespective of antifungal regimen ${ }^{185}$. To date, two randomized studies have directly compared an echinocandin with an azole for initial therapy of candidaemia. A 2007 randomized trial demonstrated that overall response rates were numerically lower with fluconazole $(60 \%)$ than with anidulafungin $(76 \%)^{186}$, and a 2016 larger study yielded highly similar outcomes for isavuconazole $(60 \%)$ compared with caspofungin $(71 \%)^{190}$.

C. parapsilosis is a low-virulence organism that consistently demonstrates higher MICs for echinocandins than most other Candida spp. However, in contrast to patients with candidaemia caused by other Candida spp. with acquired resistance and high MICs, observational data from Spain and the United States and combined data from several clinical trials suggest no difference in outcome among patients with candidaemia due to $C$. parapsilosis between those who received initial treatment with an echinocandin and those who received other regimens ${ }^{191-193}$. Thus, initial therapy with an echinocandin is appropriate for most patients with C. parapsilosis infection.

Azoles. The azoles are a group of antifungal compounds with broad activity against most Candida spp. They are generally well tolerated, have predictable drug-drug interactions and can be administered as systemic agents either intravenously or orally. Azole antifungals inhibit lanosterol 14a-demethylase, a key enzyme in the formation of ergosterol, which is a major component of the fungal cell membrane. Fluconazole was one of the drugs of choice for candidaemia until the echinocandins became generally available in 2003 , and now is a second-line agent for initial therapy in most circumstances. Of note, azole therapy is preferred in specific clinical situations, such as endophthalmitis, meningitis and urinary tract candidiasis, in which echinocandins do not reach sufficient concentrations, as echinocandins do not sufficiently pass the blood-brain barrier and are not excreted into the urine. Voriconazole is an effective alternative for invasive candidiasis but offers little substantial advantage over therapy with fluconazole in most instances ${ }^{187}$.

In parts of the world with limited resources and low prevalence of azole resistance, fluconazole remains the standard initial treatment for patients with invasive candidiasis. However, fluconazole should be considered as first-line therapy with caution in light of recent data pertaining to increasing fluconazole resistance, particularly among regions where C. auris is prevalent ${ }^{7}$. Haemodynamically stable patients who have had no previous exposure to azoles and who do not belong to a group at high risk of C. glabrata infection (such as elderly patients or patients with diabetes mellitus and/or cancer), in settings where access to echinocandins is limited and/or parenteral therapy is impractical, are reasonable candidates for oral fluconazole first-line therapy ${ }^{106}$.

\section{Step-down therapy}

After the patient's conditions are stabilized, stepdown therapy is commonly prescribed and supported by clinical data ${ }^{188}$. This transition is generally made within 3-7 days from initial therapy, but the timing 
varies substantially on the basis of both the patient's response and the clinician's preference. An oral azole (usually fluconazole) is the first-line agent for stepdown therapy, although there must be no evidence of azole resistance based on species determination or antifungal susceptibility data. Non-comparative trials have assessed outcomes among patients with candidaemia caused by azole-susceptible Candida spp. who received an echinocandin for at least 5 days and, once their conditions had stabilized and blood cultures were negative, switched to step-down therapy with an oral azole (fluconazole or voriconazole) or continued the echinocandin regimen at the same dosage ${ }^{188,194}$. No differences in 30-day survival or mycological cure rate were observed between patients who continued to receive echinocandin as step-down therapy and those who received an oral azole $\mathrm{e}^{188,194}$.

Voriconazole is an acceptable step-down agent for patients who are ready for transition from initial therapy to step-down oral therapy and who have fluconazoleresistant isolates such as C. krusei and Candida guilliermondii, and potentially C. glabrata. Posaconazole and isavuconazole also have excellent in vitro activity against C. krusei, C. guilliermondii and C. glabrata, but there are few clinical data to support their routine use.

\section{Duration of treatment}

The duration of antifungal treatment is generally determined by the individual clinical and mycological response to therapy. Among patients with candidaemia, follow-up blood cultures at least every other day until documented clearance of Candida spp. from the bloodstream are essential to establish the appropriate duration of treatment. In the absence of organ involvement, the duration of systemic (intravenous or oral) antifungal therapy should be 14 days after clearance of Candida spp. from the bloodstream (if the blood culture was initially positive) and resolution of all signs of infection, such as fever and hypotension ${ }^{106,107}$.

\section{Deep-seated infections}

For patients with less common manifestations of deep-seated invasive candidiasis, treatment decisions are based more on experience and/or anecdote rather than prospective data. For osteoarticular involvement, chronic hepatosplenic candidiasis and intra-abdominal candidiasis, there are no prospective treatment data, and most clinicians take a modified approach to therapy, such as initial therapy with echinocandins until there is a favourable clinical and/or radiographic response, and then transition to an oral azole (such as fluconazole) for several months ${ }^{106,107}$. The duration of treatment in these situations depends on the site of involvement and ranges from several weeks to 6-12 months. Occasionally, patients require chronic, long-term antifungal suppressive therapy for refractory infections such as prosthetic valve endocarditis, chronic prosthetic joint infection or infected intravascular devices that cannot be removed or replaced (for example, left ventricular assist devices and intracardiac pacemakers or defibrillators) $)^{106,107}$.

\section{Drug resistance}

Echinocandin-resistant isolates, especially C. glabrata isolates, are frequently also fluconazole resistant, and resistance to both classes of antifungals seems to be associated with worse clinical outcomes ${ }^{14}$. The recent emergence of MDR C. auris poses especially challenging questions relating to effective therapy. As there are currently no prospective data to guide treatment decisions in these situations, most clinicians favour a lipid formulation of AmB for treatment of patients with invasive candidiasis due to proven or suspected MDR Candida spp. strains until more data become available.

$\mathrm{AmB}$ is an antifungal from the polyene class that binds to ergosterol on the fungal membrane and has broad activity against all Candida spp. but not against Candida lusitaniae, which is frequently resistant. AmB has historically been associated with severe adverse effects, particularly nephrotoxicity. Lipid formulations of $\mathrm{AmB}$ are preferred owing to their efficacy ${ }^{195}$, lower incidence of infusion-related adverse effects and reduced risk of nephrotoxicity compared with $\mathrm{AmB}$ deoxycholate. An AmB formulation should be taken into account in patients with a history of intolerance to echinocandins and/or azoles, when the infection is refractory to other therapy, in isolates (such as C. auris) with suspected or proven resistance to other agents, in some deep-seated infections such as valvular endocarditis and meningoencephalitis and for direct intraocular administration for endophthalmitis.

\section{Quality of life}

There have been no quality of life (QOL) studies among survivors of invasive candidiasis. The most objective QOL measure associated with invasive candidiasis is survival at 30 days. Most studies suggest that this figure should be $>60 \%$ in large groups of patients ${ }^{185}$. The majority of survivors of uncomplicated candidaemia have no residual effects from the infection, with the exception of patients with ocular involvement associated with candidaemia, which is a major morbidity that can lead to permanent visual impairment including blindness if not recognized early and treated aggressively ${ }^{192}$. Most individuals resume prior activities without effect on QOL, and the risk of recurrent disease is quite uncommon $(<10 \%)^{186-190}$. When relapse does occur, it is most often associated with persistence of an endovascular focus, such as a retained implantable central venous catheter, prosthetic heart valve or implantable defibrillator. Less commonly, an infected retained prosthetic device such as orthopaedic hardware may be the cause of persistent or recurring disease. Rarely, a persistent intra-abdominal or intra-thoracic source, such as an incompletely drained abscess, can be a source of persistent infection. In each of these instances, QOL can be substantially affected by the morbidity associated with a chronic persistent infection, including the need for additional surgical procedures, such as cardiac valve excision and replacement, removal and replacement of a prosthetic orthopaedic joint, intraocular debridement or intra-abdominal surgical drainage and/or debridement. 
Administering systemic antifungal therapy to patients on a long-term basis is often challenging. Long-term therapy is expensive and inconvenient and usually requires at least once-daily administration; additionally, it is also associated with potential drug-related intolerance (for example, nausea and vomiting), drug-drug interactions (for example, voriconazole and fluconazole interaction with the antibiotic rifampin and immune suppressants cyclosporine and tacrolimus) and substantial direct drug-associated toxicity (for example, renal impairment, liver function abnormalities and rash). Most of these drug effects, for example, elevated liver function tests associated with use of fluconazole or voriconazole, are reversible upon discontinuation of the compound. Others, such as AmB-related renal impairment due to long-term treatment, could be irreversible.

\section{Outlook}

Invasive candidiasis is the most common fungal disease among hospitalized patients and has continued to be a major cause of mortality. Although diagnostic tools and management strategies have improved, the changing epidemiology towards non-albicans Candida spp. and the emerging resistance to antifungal drugs are of increasing concern. The prevalence of non-albicans Candida spp. has been steadily increasing in many parts of the world. The selective pressure of antifungal use has further driven the emergence of species that are typically less drug sensitive than C. albicans, such as C. glabrata (in settings with heavy fluconazole use) and C. parapsilosis (in settings with heavy use of echinocandins) $)^{12,196}$. In addition, acquired echinocandin resistance has been reported with increasing frequency, particularly in C. glabrata ${ }^{12}$. The recent emergence of MDR C. auris as a cause of nosocomial outbreaks with rapid spread is worrisome $\mathrm{e}^{7-9}$. Owing to its capability to persist in hospital environments and cause lengthy outbreaks, its propensity to acquire resistance mechanisms and its potential misidentification by current diagnostic systems, $C$. auris is a potential global threat. Outstanding questions are whether $C$. auris outbreaks will be contained to settings with suboptimal Candida spp. identification and infection control, or whether this and other MDR species will spread and mark the beginning of a new era. In addition, changes in species distribution will drive changes in future treatment recommendations, as the differential susceptibilities of the various Candida spp. to azoles and echinocandins influence treatment. At this time, there is a crucial need for novel antifungal agents with new mechanisms of action to ensure successful treatment of Candida spp. that are resistant to azoles and echinocandins.

\section{Diagnostics}

Non-culture-based, PCR-based diagnostic techniques have been rapidly evolving ${ }^{144,153}$ and will markedly affect the diagnostic work-up of patients within the next 5 years. By contrast, biomarkers, including Candida spp. mannan and $\beta$-D-glucan and antimannan antibodies, have had a variable and somewhat disappointing performance. Their specificity for candidaemia or deepseated candidiasis usually is limited, with many patients with Candida spp. colonization but without infection having positive test results ${ }^{131}$, whereas their sensitivity, and hence their negative predictive value, can be inadequate in selected populations at very high risk of candidaemia ${ }^{4,131}$. Unless new biomarkers are identified, diagnostic strategies will likely shift towards direct, DNA-based Candida spp. detection.

At a time when most attention has been focused on detecting candidaemia, the diagnosis and management of deep-seated (for example, intra-abdominal) non-candidaemic infections require further clinical studies. Data suggesting an important role for plasma PCR in detecting deep-seated invasive candidiasis are promising ${ }^{197}$.

\section{Treatment}

On the basis of a growing body of evidence, the management of patients with invasive candidiasis has changed markedly since the 2000s. Source control and early initiation of appropriate antifungal therapy have been consistently reported as major determinants of survival ${ }^{183,184}$, and an emerging body of evidence now supports the superiority of echinocandins for primary treatment of invasive candidiasis ${ }^{185,186,190}$.

Few studies are available to support recommendations on the optimal step-down therapy from echinocandins to azoles or the total duration of therapy. Whereas early initial therapy with echinocandins is associated with reduced mortality, step-down therapy to an azole once the patient is clinically stable and Candida spp. have been cleared from the bloodstream seems to be effective as early as 3-7 days into initial therapy ${ }^{188}$. Strategy studies should prospectively identify optimal step-down protocols as well as explore further limitations of the total duration of antifungal treatment in selected patient groups.

Studies in patients with culture-proven invasive candidiasis have identified early appropriate treatment as a major driver of outcomes. Thus, it is plausible that early, presumptive treatment of patients with possible invasive candidiasis is also beneficial. However, such strategies have not been successfully validated by prospective studies. Trials on empirical or presumptive treatment of high-risk patients in the ICU have been disappointing. Even studies in which treatment decisions were refined by taking into account parameters such as Candida spp. colonization and serum biomarkers have failed to demonstrate a reduction of mortality or length of stay ${ }^{131,167}$. The failure to reliably identify individuals with yet-undetected invasive candidiasis is partly explained by the low prevalence of invasive candidiasis, even in the ICU, and by the very low positive predictive value of prediction rules applying clinical risk factors and biomarkers. Thus, such rules might at best be an additional component in the evaluation of a patient's risk of developing candidiasis, rather than a decision-making tool ${ }^{4}$.

\section{Prevention}

The challenges in Candida spp. vaccine development are highly specific to the diverse manifestations of the disease and to the host defence pathways involved, which demand a cellular vaccine target rather than protective 
antibodies, as neutrophil, monocyte and/or macrophage functions are the major components of the anti-Candida spp. host defence. Recurrent vulvovaginal candidiasis, the most evident target for vaccine development in view of its chronic and recurrent nature, would require a vaccine aimed at improving $\mathrm{T}_{\mathrm{H}} 17$ cell pathway-driven mucosal immunity and at protecting against mucosal invasion of Candida spp. rather than colonization. The first Candida spp. vaccine candidate in humans, which targeted agglutinin-like protein 3, was recently tested safely in a phase I clinical trial and was efficacious in reducing episodes of vaginitis in women with recurrent vulvovaginal candidiasis in a phase II clinical trial ${ }^{36-38}$. Mechanistically, vaccine protection during invasive candidiasis is mediated by enhanced neutrophil recruitment in infected tissue ${ }^{198}$. For patients at risk of candidaemia and invasive candidiasis, a vaccine should preferably target $\mathrm{T}_{\mathrm{H}} 1$ cell pathways. Vaccination will likely not be aimed at the general, healthy population but at specific patients at immediate risk, such as candidates for surgery or for stem cell or solid organ transplant. This poses an additional challenge for vaccine efficacy in patients with underlying diseases and immune defects.

Antifungal prophylaxis for selected patients at high risk in the ICU seems appropriate, but studies have shown that such treatment did not improve patient survival $^{122,167,168}$. Future studies should aim to further identify risk factors to select individual patients who will benefit most from prophylaxis. As sets of clinical risk factors have been explored exhaustively with limited success ${ }^{168}$, the identification of individual immunogenetic traits rendering individuals prone to acquiring invasive candidiasis will open new avenues for individualized, targeted prophylaxis and treatment.

\section{Determinants for personalized therapy}

Specific cytokine pathways and gene polymorphisms have now been identified that are associated with a 10 -fold to 20 -fold increase in susceptibility to invasive candidiasis among patients in the ICU ${ }^{199}$. Within the next 5-10 years, the rapid development of fungal immunogenetics will likely generate clinical tools to select the highest-risk patients for Candida spp. vaccination or prophylactic antifungal treatment upon admission to the ICU or transplant unit. Similarly, immunogenetic traits have been identified that are associated with persistent candidaemia and unfavourable outcomes in patients in the ICU once they have acquired invasive candidiasis ${ }^{97,199}$. Such knowledge will likely steer the use of adjunctive immunotherapy but also long-term antifungal treatment or intensified monitoring.

In conclusion, new antifungal treatment regimens have led to lower mortality among patients with invasive candidiasis. Further studies should focus on earlier diagnosis by non-culture-based molecular tests and on early intervention strategies based on combined clinical prediction rules, molecular tests and personalized, immunogenetics-based risk profiles.
1. Magill, S. S. et al. Multistate point-prevalence survey of health care-associated infections. N. Engl. J. Med. 370, 1198-1208 (2014).

2. McCarty, T. P. \& Pappas, P. G. Invasive candidiasis. J. Infect. Dis. Clin. North Am. 30, 103-124 (2016).

3. Pappas, P. G. et al. A prospective observational study of candidemia: epidemiology, therapy, and influences on mortality in hospitalized adult and pediatric patients. Clin. Infect. Dis. 37, 634-643 (2003).

4. Kullberg, B. J. \& Arendrup, M. C. Invasive candidiasis N. Engl. J. Med. 373, 1445-1456 (2015).

5. Wisplinghoff, H. et al. Nosocomial bloodstream infections in US hospitals: analysis of 24,179 cases from a prospective nationwide surveillance study. Clin. Infect. Dis. 39, 309-176 (2004).

6. Cleveland, A. A. et al. Declining incidence of candidemia and the shifting epidemiology of Candida resistance in two US metropolitan areas, 2008-2013. results from population-based surveillance. PLOS ONE 10, e0120452 (2015).

This is a population-based review documenting the worldwide trend of changing epidemiology, emergence of multidrug resistance owing to echinocandin-resistant C. glabrata and the importance of stringent infection control.

7. Chowdhary, A., Sharma, C. \& Meis, J. F. Candida auris: A rapidly emerging cause of hospital-acquired multidrug-resistant fungal infections globally. PLoS Pathog. 13, e1006290 (2017). This is the first review to describe the worldwide emergence of $C$. auris.

8. Lockhart, S. R. et al. Simultaneous emergence of multidrug-resistant Candida auris on 3 continents confirmed by whole-genome sequencing and epidemiological analyses. Clin. Infect. Dis. 64 , 134-140 (2017)

9. Clancy, C. J. \& Nguyen, M. H. Emergence of Candida auris: an international call to arms. Clin. Infect. Dis. 64, 141-143 (2017).

10. Castanheira, M., Messer, S. A., Rhomberg, P. R. $\&$ Pfaller, M. A. Antifungal susceptibility patterns of a global collection of fungal isolates: results of the SENTRy antifungal surveillance program Diagn. Microbiol. Infect. Dis. 85, 200-204 (2016).
11. Pfaller, M. A., Moet, G. J., Messer, S. A., Jones, R. N. $\&$ Castanheira, M. Geographic variations in species distribution and echinocandin and azole antifungal resistance rates among Candida bloodstream infection isolates: report from the SENTRY antimicrobial surveillance program J. Clin. Microbiol. 49, 396-399 (2011).

12. Vallabhaneni, S. et al. Epidemiology and risk factors for echinocandin nonsusceptible Candida glabrata bloodstream infections: data from a large multisite population-based candidemia surveillance program, 2008-2014. Open Forum Infect. Dis. 2, ofv 163 (2015).

13. Arendrup, M. C. \& Perlin, D. S. Echinocandin resistance: an emerging clinical problem? Curr. Opin Infect. Dis. 27, 484-492 (2014).

14. Alexander, B. D. et al. Increasing echinocandin resistance in Candida glabrata: clinical failure correlates with presence of FKS mutations and elevated minimum inhibitory concentrations. Clin. Infect. Dis. 56, 1724-1732 (2013).

15. Vincent, J. L. et al. International study of the prevalence and outcomes of infection in intensive care units. JAMA 302, 2323-2329 (2009).

16. Playford, E. G. et al. Assessment of clinical risk predictive rules for invasive candidiasis in a prospective multicentre cohort of ICU patients. Intensive Care Med. 35, 2141-2145 (2009).

17. Ostrosky-Zeichner, L. et al. Multicenter retrospective development and validation of a clinical prediction rule for nosocomial invasive candidiasis in the intensive care setting. Eur. J. Clin. Microbiol. Infect. Dis. 26, 271-276 (2007).

18. Strollo, S., Lionakis, M. S., Adjemian, J., Steiner, C. A. \& Prevots, D. R. Epidemiology of hospitalizations associated with invasive candidiasis, United States, 2002-2012. Emerg. Infect. Dis. 23, 7-13 (2016).

19. Ifrim, D. C. et al. Role of Dectin-2 for host defense against systemic infection with Candida glabrata. Infect. Immun. 82, 1064-1673 (2014).

20. Linden, J. R., Kunkel, D., Laforce-Nesbitt, S. S $\&$ Bliss, J. M. The role of galectin-3 in phagocytosis of Candida albicans and Candida parapsilosis by human neutrophils. Cell. Microbiol. 15, 1127-1142 (2013).
21. Gow, N. A., van de Veerdonk, F. L., Brown, A. J. \& Netea, M. G. Candida albicans morphogenesis and host defence: discriminating invasion from colonization. Nat. Rev. Microbiol. 10, 112-122 (2011).

22. Netea, M. G., Joosten, L. A., van der Meer, J. W. Kullberg, B. J. \& van de Veerdonk, F. L. Immune defence against Candida fungal infections. Nat. Rev Immunol. 15, 630-642 (2015).

23. Fan, D. et al. Activation of HIF-1 alpha and LL-37 by commensal bacteria inhibits Candida albicans colonization. Nat. Med. 21, 808-814 (2015).

24. Lionakis, M. S. \& Netea, M. G. Candida and host determinants of susceptibility to invasive candidiasis. PLoS Pathog. 9, e1003079 (2013).

25. Lionakis, M. S., Netea, M. G. \& Holland, S. M. Mendelian genetics of human susceptibility to fungal infection. Cold Spring Harb. Perspect. Med. 4, a019638 (2014).

26. Puel, A. et al. Inborn errors of human IL-17 immunity underlie chronic mucocutaneous candidiasis. Curr. Opin. Allergy Clin. Immunol. 12, 616-622 (2012).

27. Lo, H. J. et al. Nonfilamentous C. albicans mutants are avirulent. Cell 90, 939-949 (1997).

28. Murad, A. M. et al. NRG1 represses yeast-hypha morphogenesis and hypha-specific gene expression in Candida albicans. EMBO J. 20, 4742-4752 (2001).

29. Netea, M. G. et al. Immune sensing of Candida albicans requires cooperative recognition of mannans and glucans by lectin and Toll-like receptors. J. Clin. Invest. 116, 1642-1650 (2006)

30. Marakalala, M. J. et al. Differential adaptation of Candida albicans in vivo modulates immune recognition by dectin-1. PLoS Pathog. 9, e 1003315 (2013).

31. Wheeler, R. T., Kombe, D., Agarwala, S. D., Fink, G. R. Dynamic, morphotype-specific Candida albicans betaglucan exposure during infection and drug treatment. PLoS Pathog. 4, e1000227 (2008)

32. Felk, A. et al. Candida albicans hyphal formation and the expression of the Efg 1-regulated proteinases Sap4 to Sap 6 are required for the invasion of parenchymal organs. Infect. Immun. 70, 3689-3700 (2002).

33. Gabrielli, E. et al. In vivo induction of neutrophil chemotaxis by secretory aspartyl proteinases of Candida albicans. Virulence 7, 819-825 (2016). 
34. Moyes, D. L. et al. Candidalysin is a fungal peptide toxin critical for mucosal infection. Nature 532 64-68 (2016)

35. Sheppard, D. C. \& Filler, S. G. Host cell invasion by medically important fungi. Cold Spring Harb. Perspect. Med. 5, a019687 (2014)

36. Phan, Q. T. et al. Als3 is a Candida albicans invasin that binds to cadherins and induces endocytosis by host cells. PLoS Biol. 5, e64 (2007).

37. Schmidt, C. S. et al. NDV-3, a recombinant alumadjuvanted vaccine for Candida and Staphylococcus aureus, is safe and immunogenic in healthy adults. Vaccine 30, 7594-7600 (2012)

38. Edwards, J. E. Jr et al. A fungal immunotherapeutic vaccine (NDV-3A) for treatment of recurrent vulvovaginal candidiasis; a phase 2 randomized, double-blind, placebo controlled trial. Clin. Infect. Dis. https://doi.org/10.1093/cid/ciy185 (2018).

39. Desai, J. V., Mitchell, A. P. \& Andes, D. R. Fungal biofilms, drug resistance, and recurrent infection. Cold Spring Harb. Perspect. Med. 4, a019729 (2014).

40. Nobile, C. J. et al. A recently evolved transcriptiona network controls biofilm development in Candida albicans. Cell 148, 126-138 (2012).

41. Jensen, R. H., Justesen, U. S., Rewes, A., Perlin, D. S. $\&$ Arendrup, M. C. Echinocandin failure case due to a previously unreported FKS1 mutation in Candida krusei. Antimicrob. Agents Chemother. 58 3550-3552 (2014).

42. Arendrup, M. C. et al. Differential in vivo activities of anidulafungin, caspofungin, and micafungin against Candida glabrata isolates with and without FKS resistance mutations. Antimicrob. Agents Chemother. 56, 2435-2442 (2012)

43. Morio, F., Jensen, R. H., Le Pape, P. \& Arendrup, M. C Molecular basis of antifungal drug resistance in yeasts. Int. J. Antimicrob. Agents. 50, 599-606 (2017).

44. Jensen, R. H. et al. Stepwise emergence of azole echinocandin and amphotericin B multidrug resistance in vivo in Candida albicans orchestrated by multiple genetic alterations. J. Antimicrob. Chemother. 70, 2551-2555 (2015)

45. Healey, K. R. et al. Prevalent mutator genotype identified in fungal pathogen Candida glabrata promotes multi-drug resistance. Nat. Commun. 7 , 11128 (2016)

This is the first description of the potential role of the DNA mismatch repair gene $M S H 2$ in facilitating drug resistance in C. glabrata. In vitro and in vivo (mouse model) experimentation demonstrate an increased rate of drug resistance in MSH2 mutants and that MSH2 mutants are found among clinical isolates. The potential role in clinical epidemiology remains to be clarified.

46. Farmakiotis, D., Tarrand, J. J. \& Kontoyiannis, D. P. Drug-resistant Candida glabrata infection in cancer patients. Emerg. Infect. Dis. 20, 1833-1840 (2014)

47. Brown, G. D. Innate antifungal immunity: the key role of phagocytes. Annu. Rev. Immunol. 29, 1-21 (2011).

48. Erwig, L. P. \& Gow, N. A. Interactions of fungal pathogens with phagocytes. Nat. Rev. Microbiol. 14, 163-176 (2016)

49. Hardison, S. E. \& Brown, G. D. C-Type lectin receptors orchestrate antifungal immunity. Nat. Immunol. 13 817-822 (2012)

50. Jaeger, M. et al. The RIG-I-like helicase receptor MDA5 (IFIH1) is involved in the host defense against Candida infections. Eur. J. Clin. Microbiol. Infect. Dis. 34, 963-974 (2015).

51. LeibundGut-Landmann, S., Wuthrich, M. \& Hohl, T. M. Immunity to fungi. Curr. Opin. Immunol. 24, 449-458 (2012).

52. Lionakis, M. S. New insights into innate immune control of systemic candidiasis. Med. Mycol. 52, 555-564 (2014).

53. Netea, M. G., Brown, G. D., Kullberg, B. J. $\ltimes$ Gow, N. A. An integrated model of the recognition of Candida albicans by the innate immune system. Nat. Rev. Microbiol. 6, 67-78 (2008).

54. Gringhuis, S. I. et al. Dectin-1 is an extracellular pathogen sensor for the induction and processing of IL- 1 beta via a noncanonical caspase- 8 inflammasome. Nat. Immunol. 13, 246-254 (2012).

55. Gross, O. et al. Syk kinase signalling couples to the Nlrp3 inflammasome for anti-fungal host defence. Nature 459, 433-436 (2009).

56. Joly, S. et al. Cutting edge: NIrp10 is essential for protective antifungal adaptive immunity against Candida albicans. J. Immunol. 189, 4713-4717 (2012).
57. Cheng, S. C. et al. Complement plays a central role in Candida albicans-induced cytokine production by human PBMCs. Eur. J. Immunol. 42, 993-1004 (2012).

58. Jouault, T. et al. Specific recognition of Candida albicans by macrophages requires galectin- 3 to discriminate Saccharomyces cerevisiae and needs association with TLR2 for signaling. J. Immunol. 177 4679-4687 (2006)

59. Zhu, L. L. et al. C-Type lectin receptors Dectin-3 and Dectin-2 form a heterodimeric pattern-recognition receptor for host defense against fungal infection. Immunity 39, 324-334 (2013).

60. Dennehy, K. M., Willment, J. A., Williams, D. L. \& Brown, G. D. Reciprocal regulation of IL-23 and IL-12 following co-activation of Dectin-1 and TLR signaling pathways. Eur. J. Immunol. 39, 1379-1386 (2009).

61. Deng, Z. et al. Tyrosine phosphatase SHP-2 mediates C-type lectin receptor-induced activation of the kinase Syk and anti-fungal TH17 responses. Nat. Immunol. 16, 642-652 (2015).

62. Gorjestani, S. et al. Phospholipase Cgamma2 (PLCgamma2) is key component in Dectin-2 signaling pathway, mediating anti-fungal innate immune responses. J. Biol. Chem. 286, 43651-43659 (2011).

63. Strasser, D. et al. Syk kinase-coupled C-type lectin receptors engage protein kinase $\mathrm{C}$-sigma to elicit Card9 adaptor-mediated innate immunity. Immunity 36, 32-42 (2012)

64. Jia, X. M. et al. CARD9 mediates Dectin-1-induced ERK activation by linking Ras-GRF1 to H-Ras for antifungal immunity. J. Exp. Med. 211, 2307-2321 (2014)

65. Drummond, R. A. \& Lionakis, M. S. Mechanistic insights into the role of C-type lectin receptor/CARD9 signaling in human antifungal immunity. Front. Cell. Infect. Microbiol. 6, 39 (2016).

66. Lanternier, F. et al. Inherited CARD9 deficiency in otherwise healthy children and adults with Candida species-induced meningoencephalitis, colitis, or both J. Allergy Clin. Immunol. 135, 1558-1568.e2 (2015).

67. von Bernuth, H. et al. Pyogenic bacterial infections in humans with MyD88 deficiency. Science 321 691-696 (2008)

68. Ross, S. C. \& Densen, P. Complement deficiency states and infection: epidemiology, pathogenesis and consequences of neisserial and other infections in an immune deficiency. Medicine 63, 243-273 (1984).

69. Glocker $\mathrm{O}$ O et al. Homozygous CARD9 mutation in a family with susceptibility to fungal infections N. Engl. J. Med. 361, 1727-1735 (2009). This study is the first to describe CARD9 deficiency as a primary immunodeficiency that results in fungal-specific infection susceptibility to mucosal candidiasis and invasive candidiasis that specifically involves the central nervous system.

70. Drummond, R. A. et al. CARD9-dependent neutroph recruitment protects against fungal invasion of the central nervous system. PLoS Pathog. 11, e1005293 (2015).

71. Ferwerda, B. et al. Human dectin-1 deficiency and mucocutaneous fungal infections. N. Engl. J. Med. 361, 1760-1767 (2009).

72. Romani, L et al. An immunoregulatory role for neutrophils in CD4 + Thelper subset selection in mice with candidiasis. J. Immunol. 158, 2356-2362 (1997)

73. Uzun, O., Ascioglu, S., Anaissie, E. J. \& Rex, J. H. Risk factors and predictors of outcome in patients with cancer and breakthrough candidemia. Clin. Infect. Dis. 32, 1713-1717 (2001)

74. Lionakis, M. S., Lim, J. K., Lee, C. C. \& Murphy, P. M Organ-specific innate immune responses in a mouse model of candidiasis. J. Innate Immun. 3, 180-199 (2011).

75. Reeves, E. P. et al. Killing activity of neutrophils is mediated through activation of proteases by $\mathrm{K}^{+}$flux. Nature 416, 291-297 (2002).

76. Segal, B. H., Leto, T. L., Gallin, J. I., Malech, H. L. \& Holland, S. M. Genetic, biochemical, and clinical features of chronic granulomatous disease. Medicine 79, 170-200 (2000).

77. Lehrer, R. I. \& Cline, M. J. Leukocyte myeloperoxidase deficiency and disseminated candidiasis: the role of myeloperoxidase in resistance to Candida infection. J. Clin. Invest. 48, 1478-1488 (1969).

78. Greenblatt, M. B., Aliprantis, A., Hu, B.

$\&$ Glimcher, L. H. Calcineurin regulates innate antifungal immunity in neutrophils. J. Exp. Med. 207 923-931 (2010).
79. Li, X et al. The beta-glucan receptor Dectin-1 activates the integrin Mac-1 in neutrophils via Vav protein signaling to promote Candida albicans clearance. Cell Host Microbe 10, 603-615 (2011).

80. Lehrer, R. I. Measurement of candidacidal activity of specific leukocyte types in mixed cell populations I. Normal, myeloperoxidase-deficient, and chronic granulomatous disease neutrophils. Infect. Immun. 2, 42-47 (1970)

81. Swamydas, M. et al. CXCR1-mediated neutrophil degranulation and fungal killing promote Candida clearance and host survival. Sci. Transl Med. 8 $322 \mathrm{ra3} 10$ (2016)

82. Wirnsberger, G. et al. Jagunal homolog 1 is a critical regulator of neutrophil function in fungal host defense. Nat. Genet. 46, 1028-1033 (2014).

83. Gazendam, R. P., van de Geer, A., Roos, D. van den Berg, T. K. \& Kuijpers, T. W. How neutrophils kill fungi. Immunol. Rev. 273, 299-311 (2016).

84. Gazendam, R. P. et al. Two independent killing mechanisms of Candida albicans by human neutrophils: evidence from innate immunity defects. Blood 124, 590-597 (2014).

85. Legrand, F. et al. Adjuvant corticosteroid therapy for chronic disseminated candidiasis. Clin. Infect. Dis. 46, 696-702 (2008)

86. Tomashefski, J. F. Jr \& Abramowsky, C. R. Candidaassociated renal papillary necrosis. Am. J. Clin. Pathol 75, 190-194 (1981)

87. Lionakis, M. S. et al. CX3CR1-dependent renal macrophage survival promotes Candida control and host survival. J. Clin. Invest. 123, 5035-5051 (2013).

This study is the first to describe the role of $\mathrm{CX}_{3} \mathrm{C}$-chemokine receptor 1 ( $\left.\mathrm{CX}_{3} \mathrm{CR} 1\right)$ expressing resident macrophages in host defence against invasive candidiasis and to identify the dysfunctional CX3CR1-M280 allele as a risk factor for candidaemia and disseminated candidiasis in humans.

88. Kanayama, M. et al. Autophagy enhances NFkappaB activity in specific tissue macrophages by sequestering A20 to boost antifungal immunity. Nat. Commun. 6 5779 (2015).

89. Ngo, L. Y. et al. Inflammatory monocytes mediate early and organ-specific innate defense during systemic candidiasis. J. Infect. Dis. 209, 109-119 (2014).

90. Quintin, J. et al. Candida albicans infection affords protection against reinfection via functional reprogramming of monocytes. Cell Host Microbe 12 , 223-232 (2012)

91. Break, T. J. et al. Batf3-dependent CD103+ dendritic cell accumulation is dispensable for mucosal and systemic antifungal host defense. Virulence 7 , 826-835 (2016)

92. del Fresno, C. et al. Interferon-beta production via Dectin-1-Syk-IRF5 signaling in dendritic cells is crucial for immunity to C. albicans. Immunity 38, 1176-1186 (2013).

93. Netea, M. G. et al. Human dendritic cells are less potent at killing Candida albicans than both monocytes and macrophages. Microbes Infect. 6, 985-989 (2004)

94. Whitney, P. G. et al. Syk signaling in dendritic cells orchestrates innate resistance to systemic fungal infection. PLoS Pathog. 10, e1004276 (2014).

95. Bar, E., Whitney, P. G., Moor, K., Reis e Sousa, C. \& LeibundGut-Landmann, S. IL-17 regulates systemic fungal immunity by controlling the functional competence of NK cells. Immunity 40, 117-127 (2014).

96. Wan, L. et al. Effect of Granulocyte-macrophage colony-stimulating factor on prevention and treatment of invasive fungal disease in recipients of allogeneic stem-cell transplantation: a prospective multicenter randomized phase IV Trial. J. Clin. Oncol. 33, 3999-4006 (2015)

97. Johnson, M. D. et al. Cytokine gene polymorphisms and the outcome of invasive candidiasis: a prospective cohort study. Clin. Infect. Dis. 54, 502-510 (2012).

98. Kumar, V. et al. Immunochip SNP array identifies novel genetic variants conferring susceptibility to candidaemia. Nat. Commun. 5, 4675 (2014). This study is the first genome-wide analysis of genetic variants that may confer susceptibility to candidaemia, and it identifies new immunogenetic risk factors for the infection in the ICU.

99. Plantinga, T. S. et al. Toll-like receptor 1 polymorphisms increase susceptibility to candidemia. J. Infect. Dis. 205, 934-943 (2012). 
100. Smeekens, S. P. et al. Functional genomics identifies type I interferon pathway as central for host defense against Candida albicans. Nat. Commun. 4, 1342 (2013).

101. Wojtowicz, A. et al. Polymorphisms in tumor necrosis factor-alpha increase susceptibility to intra-abdomina Candida infection in high-risk surgical ICU patients* Crit. Care Med. 42, e304-e308 (2014).

102. Khanna, N. et al. Host response to fungal infections how immunology and host genetics could help to identify and treat patients at risk. Swiss Med. Wkly 146, w14350 (2016)

103. Morrell, M., Fraser, V. J. \& Kollef, M. H. Delaying the empiric treatment of candida bloodstream infection until positive blood culture results are obtained: a potential risk factor for hospital mortality. Antimicrob. Agents Chemother. 49, 3640-3645 (2005).

104. Garey, K. W. et al. Time to initiation of fluconazole therapy impacts mortality in patients with candidemia: a multi-institutional study. Clin. Infect. Dis. 43, 25-31 (2006).

105. Oude Lashof, A. M. L. et al. Ocular manifestations of candidemia. Clin. Infect. Dis. 53, 262-268 (2011)

106. Pappas, P. G. et al. Clinical Practice Guideline for the Management of Candidiasis: update by the Infectious Diseases Society of America. Clin. Infect. Dis. 62 , 1-50 (2016) This is the most recent version of international treatment guidelines for candidaemia and invasive candidiasis.

107. Cornely, O. A. et al. ESCMID guideline for the diagnosis and management of Candida diseases 2012: non-neutropenic adult patients. Clin. Microbiol. Infect. 18, 19-37 (2012)

108. Clancy, C. J. \& Nguyen, M. H. Finding the "missing $50 \%$ " of invasive candidiasis: how nonculture diagnostics will improve understanding of disease spectrum and transform patient care. Clin. Infect. Dis. 56, 1284-1292 (2013).

This is an excellent overview of the strengths and limitations for current diagnostics.

109. Cuenca-Estrella, M. et al. ESCMID* guideline for the diagnosis and management of Candida diseases 2012: diagnostic procedures. Clin. Microbiol. Infect. 18, 9-18 (2012)

110. Arendrup, M. C. et al. ECIL-3 classical diagnostic procedures for the diagnosis of invasive fungal diseases in patients with leukaemia. Bone Marrow Transplant. 47, 1030-1045 (2012).

This is a European guideline on classical diagnostics; it summarizes the diagnostic options and, importantly, how to improve the sensitivity of the classical techniques.

111. Pfeiffer, C. D. et al. Quantitation of Candida CFU in initial positive blood cultures. J. Clin. Microbiol. 49, 2879-2883 (2011)

112. Arendrup, M. C. et al. Diagnostic issues, clinical characteristics, and outcomes for patients with fungemia. J. Clin. Microbiol. 49, 3300-3308 (2011).

113. Baron, E. J. O. Melvin, P. Yagupsky, P., David, F. \& Wilson, D.M. Cumitech 1C, Blood Cultures IV (American Society for Microbiology, 2005).

114. Horvath, L. et al. Direct comparison of the BACTEC 9240 and BacT/ALERT 3D automated blood culture systems for Candida growth detection. J. Clin. Microbiol. 42, 115-118 (2004).

115. Ericson, E. L., Klingspor, L., Ullberg, M. \& Ozenci, V. Clinical comparison of the Bactec Mycosis IC/F, BacT/ Alert FA, and BacT/Alert FN blood culture vials for the detection of candidemia. Diagn. Microbiol. Infect. Dis. 73, 153-156 (2012)

116. Cateau, E. et al. Impact of yeast-bacteria coinfection on the detection of Candida sp. in an automated blood culture system. Diagn. Microbiol. Infect. Dis. 72, 328-331 (2012)

117. Wey, S. B., Mori, M., Pfaller, M. A., Woolson, R. F \& Wenzel, R. P. Risk factors for hospital-acquired candidemia. A matched case-control study. Arch. Intern. Med. 149, 2349-2353 (1989).

118. Brillowska-Dabrowska, A., Bergmann, O. Jensen, I. M., Jarløv, J. O. \& Arendrup, M. C. Typing of Candida isolates from patients with invasive infection and concomitant colonization. Scand. J. Infect. Dis. 42, 109-113 (2010).

119. Jensen, R. H. et al. Posttreatment antifungal resistance among colonizing Candida isolates in Candidemia patients: results from a systematic multicenter study. Antimicrob. Agents Chemother. 60 1500-1508 (2016).

120. Lau, A. F. et al. Candida colonization as a risk marker for invasive candidiasis in mixed medical-surgical intensive care units: development and evaluation of a simple, standard protocol. J. Clin. Microbiol. 53 1324-1330 (2015)

121. Pittet, D., Monod, M., Suter, P. M., Frenk, E. \& Auckenthaler, R. Candida colonization and subsequent infections in critically ill surgical patients. Ann. Surg. 220, 751-758 (1994).

122. Timsit, J. F. et al. Empirical Micafungin treatment and survival without invasive fungal infection in adults with ICU-Acquired sepsis, candida colonization, and multiple organ failure. JAMA 316, 1555 (2016).

123. Verduyn Lunel, F. M., Donnelly, J. P., van der Lee, H. A. L., Blijlevens, N. M. A. \& Verweij, P. E. Circulating Candida-specific antimannan antibodies precede invasive candidiasis in patients undergoing myelo-ablative chemotherapy. Clin. Microbiol. Infect. 15, 380-386 (2009).

124. Sendid, B. et al. Combined detection of mannanaemia and antimannan antibodies as a strategy for the diagnosis of systemic infection caused by pathogenic Candida species. J. Med. Microbiol. 51, 433-442 (2002).

125. Mikulska, M., Calandra, T., Sanguinetti, M. Poulain, D. $\&$ Viscoli, C. The use of mannan antigen and anti-mannan antibodies in the diagnosis of invasive candidiasis: recommendations from the third European conference on infections in leukemia Crit. Care 14, R222 (2010)

126. Duettmann, W. et al. Specificity of mannan antigen and anti-mannan antibody screening in patients with haematological malignancies at risk for fungal infection. Mycoses 59, 374-378 (2016).

127. Arendrup, M. C. et al. Detection of candidaemia in patients with and without underlying haematological disease. Clin. Microbiol. Infect. 16, 855-862 (2010)

128. Oliveri, S., Trovato, L., Betta, P., Romeo, M. G $\&$ Nicoletti, G. Experience with the Platelia Candida ELISA for the diagnosis of invasive candidosis in neonatal patients. Clin. Microbiol. Infect. 14, 391-393 (2008).

129. Verduyn Lunel, F. M. et al. Detection of the Candida antigen mannan in cerebrospinal fluid specimens from patients suspected of saving Candida meningitis. J. Clin. Microbiol. 42, 867-870 (2004).

130. Biesbroek, J. M., Verduyn Lunel, F. M., Kragt, J. J. Amelink, G. J. \& Frijns, C. J. M. Culture-negative Candida meningitis diagnosed by detection of Candida mannan antigen in CSF. Neurology 81, 1555-1556 (2013).

131. León, C. et al. Contribution of Candida biomarkers and DNA detection for the diagnosis of invasive candidiasis in ICU patients with severe abdominal conditions. Crit. Care 20, 149 (2016).

132. Karageorgopoulos, D. E. et al. Accuracy of $\beta$-D-glucan for the diagnosis of Pneumocystis jirovecii pneumonia: a meta-analysis. Clin. Microbiol. Infect. 19, 39-49 (2013)

133. Karageorgopoulos, D. E. et al. $\beta$-D-glucan assay for the diagnosis of invasive fungal infections: a meta-analysis. Clin. Infect. Dis. 52, 750-770 (2011).

134. Hanson, K. E. et al. $\beta$-D-glucan surveillance with preemptive anidulafungin for invasive candidiasis in intensive care unit patients: a randomized pilot study. PLOS ONE 7, e42282 (2012).

135. Tissot, F. et al. $\beta$-Glucan antigenemia anticipates diagnosis of blood culture-negative intraabdomina candidiasis. Am. J. Respir. Crit. Care Med. 188 1100-1109 (2013).

136. Hartl, B. et al. A retrospective assessment of four antigen assays for the detection of invasive candidiasis among high-risk hospitalized patients. Myopathologia https://doi.org/10.1007/ s11046-017-0238-1 (2018).

137. Mohr, J. F. et al. Prospective survey of $(1 \rightarrow 3)$-beta-D-glucan and its relationship to invasive candidiasis in the surgical intensive care unit setting. J. Clin. Microbiol. 49, 58-61 (2011)

138. Martinez-Jimenez, M. C. et al. Combination of Candida biomarkers in patients receiving empirical antifungal therapy in a Spanish tertiary hospital: a potential role in reducing the duration of treatment. J. Antimicrob. Chemother. 70, 3107-3115 (2015).

139. Giacobbe, D. R. et al. Combined use of serum $(1,3)-\beta$-D-glucan and procalcitonin for the early differential diagnosis between candidaemia and bacteraemia in intensive care units. Crit. Care 21, 176 (2017)

140. Racil, Z. et al. Difficulties in using 1,3-\{beta $\}$-D-glucan as the screening test for the early diagnosis of invasive fungal infections in patients with haematological malignancies-high frequency of false-positive results and their analysis. J. Med Microbiol. 59, 1016-1022 (2010)

141. Fortún, J. et al. Clinical validation of a multiplex realtime PCR assay for detection of invasive candidiasis in intensive care unit patients. J. Antimicrob. Chemother 69, 3134-3141 (2014)

142. Parra-Sánchez, M. et al. Candida albicans germ-tube antibody: evaluation of a new automatic assay for diagnosing invasive Candidiasis in ICU patients. Mycopathologia 182, 645-652 (2017).

143. De Pauw, B. et al. Revised definitions of invasive funga disease from the European organization for research and treatment of cancer/invasive fungal infections cooperative group and the national institute of allergy and infectious diseases mycoses study group (EORTC/ MSG) C. Clin. Infect. Dis. 46, 1813-1821 (2008).

144. Chang, S. S. et al. Multiplex PCR system for rapid detection of pathogens in patients with presumed sepsis - a systemic review and meta-analysis. PLOS ONE 8, e62323 (2013).

145. Jordana-Lluch, E. et al. Evaluation of the broad-range $\mathrm{PCR} / \mathrm{ESI}-\mathrm{MS}$ technology in blood specimens for the molecular diagnosis of bloodstream infections. PLOS ONE 10, e0140865 (2015)

146. Desmet, S., Maertens, J., Bueselinck, K. \& Lagrou, K. Broad-range PCR/electrospray ionization time-of-flight mass spectrometry for the detection of bacteremia and fungemia in patients with neutropenic fever. J. Clin. Microbiol. 54, 2513-2520 (2016)

147. Metzgar, D. et al. The IRIDICA BAC BSI assay: rapid sensitive and culture-independent identification of bacteria and Candida in blood. PLOS ONE 11, 1-16 (2016).

148. Lewis White, P. et al. Evaluation of a commercially developed semiautomated PCR-surface-enhanced Raman scattering assay for diagnosis of invasive fungal disease. J. Clin. Microbiol. 52, 3536-3543 (2014).

149. Westh, H. et al. Multiplex real-time PCR and blood culture for identification of bloodstream pathogens in patients with suspected sepsis. Clin. Microbiol. Infect. 15, 544-551 (2009).

150. Herne, V., Nelovkov, A., Kütt, M. \& Ivanova, M. Diagnostic performance and therapeutic impact of LightCycler SeptiFast assay in patients with suspected sepsis. Eur. J. Microbiol. Immunol. 3, 68-76 (2013).

151. von Lilienfeld-Toal, M. et al. Utility of a commercially available multiplex real-time PCR assay to detect bacterial and fungal pathogens in febrile neutropenia. J. Clin. Microbiol. 47, 2405-2410 (2009).

152. Lamoth, F. et al. Multiplex blood PCR in combination with blood cultures for improvement of microbiologica documentation of infection in febrile neutropenia. J. Clin. Microbiol. 48, 3510-3516 (2010).

153. Mylonakis, E. et al. T2 magnetic resonance assay for the rapid diagnosis of candidemia in whole blood: a clinical trial. Clin. Infect. Dis. 60, 875-880 (2015).

154. Arendrup, M. C. et al. Epidemiological changes with potential implication for antifungal prescription recommendations for fungaemia: data from a nationwide fungaemia surveillance programme. Clin. Microbiol. Infect. 19, E343-E353 (2013).

155. Wang, E. et al. The ever-evolving landscape of candidaemia in patients with acute leukaemia: nonsusceptibility to caspofungin and multidrug resistance are associated with increased mortality. J. Antimicrob. Chemother. 70, 2362-2368 (2015).

156. Arendrup, M. C., Schierbeck, J., Reiter, N., Krarup, K. B. \& Andersen, J. S. Performance evaluation of the T2Candida panel in the ICU setting - data from an ongoing study (Poster). ASM Microbe 2016 (2016)

157. Walker, B., Powers-Fletcher, M. V., Schmidt, R. L. $\&$ Hanson, K. E. Cost-effectiveness analysis of multiplex PCR with magnetic resonance detection versus empiric or blood culture-directed therapy for management of suspected candidemia. J. Clin Microbiol. 54, 718-726 (2016).

158. CLSI. Reference method for broth dilution antifunga susceptibility testing of yeasts; approved standard - third edition. CLSI https://clsi.org/media/1461/ m27a3 sample.pdf (2008)

159. Arendrup, M. C. et al. EUCAST technical note on isavuconazole breakpoints for Aspergillus, itraconazole breakpoints for Candida and updates for the antifungal susceptibility testing method documents. Clin. Microbiol. Infect. 22, 571.e1-571.e4 (2016).

160. CLSI. Reference method for broth dilution antifungal susceptibility testing of yeasts; fourth informational supplement. CLSI https://clsi.org/media/1897/ m27ed4_sample.pdf(2012). 
161. Arendrup, M. C., Cuenca-Estrella, M., Lass-Flörl, C. \& Hope, W. W. Breakpoints for antifungal agents: an update from EUCAST focussing on echinocandins against Candida spp. and triazoles against Aspergillus spp. Drug Resist. Updat. 16, 81-95 (2013). This study provides a basic understanding of antifungal susceptibility testing and interpretation

162. Arendrup, M. C. \& Pfaller, M. Caspofungin Etest susceptibility testing of Candida species: risk of misclassification of susceptible isolates of $\mathrm{C}$. glabrata and C. krusei when adopting the revised CLSI caspofungin breakpoints. Antimicrob. Agents Chemother. 56, 3965-3968 (2012).

163. Astvad, K. M., Perlin, D. S., Johansen, H. K., Jensen, R. H. \& Arendrup, M. C. Evaluation of caspofungin susceptibility testing by the new Vitek 2 AST-YSO6 yeast card using a unique collection of FKS wild-type and hot spot mutant isolates, including the five most common candida species. Antimicrob. Agents Chemother. 57, 177-182 (2013).

164. Espinel-Ingroff, A. et al. Multicenter study of epidemiological cutoff values and detection of resistance in Candida spp. to anidulafungin, caspofungin, and micafungin using the sensititre yeastone colorimetric method. Antimicrob. Agents Chemother. 59, 6725-6732 (2015).

165. Eschenauer, G. et al. Real-world experience with echinocandin MICs against Candida species in a multicenter study of hospitals that routinely perform susceptibility testing of bloodstream isolates. Antimicrob. Agents Chemother. 58, 1897-1906 (2014).

166. Shorr, A. F., Chung, K., Jackson, W. L., Waterman, P. E. $\&$ Kollef, M. H. Fluconazole prophylaxis in critically ill surgical patients: a meta-analysis. Crit. Care Med. 33 , 1928-1935 (2005).

167. Pelz, R. K. et al. Double-blind placebo-controlled trial of fluconazole to prevent candidal infections in critically ill surgical patients. Ann. Surg. 233, 542-548 (2001).

168. Ostrosky-Zeichner, L. et al. MSG-01: a randomized, double-blind, placebo-controlled trial of caspofungin prophylaxis followed by preemptive therapy for invasive candidiasis in high-risk adults in the critical care setting. Clin. Infect. Dis. 58, 1219-1226 (2014).

169. Schuster. M. G. et al. Empirical fluconazole versus placebo for intensive care unit patients: a randomized trial. Ann. Intern. Med. 149, 83-90 (2008).

170. Knitsch, W. et al. A randomized, placebo-controlled trial of preemptive antifungal therapy for the prevention of invasive candidiasis following gastrointestinal surgery for intra-abdominal infections. Clin. Infect. Dis. 61, 1671-1678 (2015).

171. Montravers, P. et al. AmarCAND2 Study Group Antifungal therapy for patients with proven or suspected Candida peritonitis: Amarcand2, a prospective cohort study in French intensive care units. Clin. Microbiol. Infect. 23, 117.e1-117.e8 (2017).

172. Guillamet, C. V., Vazquez, R., Micek, S. T., Ursu, O. $£$ Kollef, M. Development and validation of a clinical prediction rule for candidemia in hospitalized patients with severe sepsis and septic shock. J. Crit. Care. 30 , 715-720 (2015)

173. Leôn, C. et al. A bedside scoring system ("Candida score") for early antifungal treatment in nonneutropenic critically ill patients with Candida colonization. Crit. Care Med. 34, 730-737 (2006).

174. Ostrosky-Zeichner, L. et al. Improvement of a clinical prediction rule for clinical trials on prophylaxis for invasive candidiasis in the intensive care unit. Mycoses 54, 46-51 (2011).

175. Luzzati, R. et al. Peripheral and total parenteral nutrition as the strongest risk factors for nosocomial candidemia in elderly patients: a matched case-control study. Mycoses 56, 664-671 (2013)

176. Brillowska-Dabrowska, A. et al. A nosocomia outbreak of Candida parapsilosis in southern Sweden verified by genotyping. Scand. J. Infect. Dis. 41, 135-142 (2009)

177. Vaz, C. et al. Microsatellite multilocus genotyping clarifies the relationship of Candida parapsilosis strains involved in a neonatal intensive care unit outbreak. Diagn. Microbiol. Infect. Dis. 71, 59-62 (2011).

178. Saxen, H. et al. Neonatal Candida parapsilosis outbreak with a high case fatality rate. Pediatr. Infect. Dis. J. 14, 776-781 (1995).

179. Sarvikivi, E. et al. Emergence of fluconazole resistance in a Candida parapsilosis strain that caused infections in a neonatal intensive care unit. J. Clin. Microbiol. 43. 2729-2735 (2005).
180. Lin, H. C. et al. Reporting an outbreak of Candida pelliculosa fungemia in a neonatal intensive care unit. J. Microbiol. Immunol. Infect. 46, 456-462 (2013).

181. Escribano, P. et al. Endemic genotypes of Candida albicans causing fungemia are frequent in the hospital. J. Clin. Microbiol. 51, 2118-2123 (2013).

182. Marcos-Zambrano, L. J. et al. Clusters of patients with candidaemia due to genotypes of Candida albicans and Candida parapsilosis: differences in frequency between hospitals. Clin. Microbiol. Infect. 21, 677-683 (2015).

183. Kollef, M., Micek, S., Hampton, N., Doherty, J. \& Kumar, A. Septic shock attributed to Candida infection: importance of empiric therapy and source control. Clin. Infect. Dis. 54,1739-1746 (2012)

184. Vergidis, P. et al. Intra-abdominal candidiasis: the importance of early source control and antifungal treatment. PLoS One 11, e0153247 (2016).

185. Andes, D. R. et al. Impact of treatment strategy on outcomes in patients with candidemia and other forms of invasive candidiasis: a patient-level quantitative review of randomized trials. Clin. Infect. Dis. $\mathbf{5 4}$ 1110-1122 (2012). This patient-level systematic review of randomized trials for candidaemia and invasive candidiasis identifies the important role of echinocandins for primary therapy.

186. Reboli, A. C. et al. Anidulafungin versus fluconazole for invasive candidiasis. N. Engl. J. Med. 356 2472-2482 (2007)

187. Kullberg, B. J. et al. Voriconazole versus a regimen of amphotericin B followed by fluconazole for candidaemia in non-neutropenic patients: a randomised non-inferiority trial. Lancet 366 , $1435-1442$ (2005)

188. Vazquez, J. et al. Evaluation of an early step-down strategy from intravenous anidulafungin to oral azole therapy for the treatment of candidemia and other forms of invasive candidiasis: results from an openlabel trial. BMC Infect. Dis. 14, 97 (2014).

189. Pappas, P. G. et al. Micafungin versus caspofungin for treatment of candidemia and other forms of invasive candidiasis. Clin. Infect. Dis. 45, 883-893 (2007).

190. Kullberg, B. J. et al. Isavuconazole versus caspofungin in the treatment of candidaemia and other invasive Candida infections: the ACTIVE trial [abstract 1239]. ECCMID https://www.aspergillus.org.uk/content isavuconazole-versus-caspofungin-treatmentcandidaemia-and-other-invasive-candida-infections (2016)

191. Fernández-Ruiz, M. et al. CANDIPOP project: GEIH-GEMICOMED (SEIMC); REIPI. Initial use of echinocandins does not negatively influence outcome in Candida parapsilosis bloodstream infection: a propensity score analysis. Clin. Infect. Dis. $\mathbf{5 8}$ 1413-1421 (2014)

192. Kontoyiannis, D. P. et al. Anidulafungin for the treatment of candidaemia caused by Candida parapsilosis: analysis of pooled data from six prospective clinical studies. Mycoses 60, 663-667 (2017).

193. Chiotos, K. et al. Comparative effectiveness of echinocandins versus fluconazole therapy for the treatment of adult candidaemia due to Candida parapsilosis: a retrospective observational cohort study of the Mycoses study group (MSG-12). J. Antimicrob. Chemother. 71, 3536-3539 (2016).

194. Kullberg, B. J. et al. Efficacy of anidulafungin in 539 patients with invasive candidiasis: a patient-level pooled analysis of six clinical trials. J. Antimicrob. Chemother 72, 2368-2377 (2017).

195. Kuse, E. R. et al. Micafungin versus liposomal amphotericin B for candidaemia and invasive candidosis: a phase III randomized double-blind trial. Lancet 369, 1519-1527 (2007).

196. Lortholary, O. et al. Recent exposure to caspofungin or fluconazole influences the epidemiology of candidemia: a prospective multicenter study involving 2,441 patients. Antimicrob. Agents Chemother. 55, 532-538 (2011).

197. Nguyen, M. H. et al. Performance of candida real-time polymerase chain reaction, $\beta$-D-glucan assay, and blood cultures in the diagnosis of invasive candidiasis. Clin. Infect. Dis. 54, 1240-1248 (2012).

198. Lin, L. et al. Th1-Th17 cells mediate protective adaptive immunity against Staphylococcus aureus and Candida albicans infection in mice. PLoS Pathog. 5, e 1000703 (2009)

199. Kullberg, B., van de Veerdonk, F. \& Netea, M. C. Immunotherapy - a potential adjunctive treatment for fungal infection. Curr. Opin. Infect. Dis. 27, 511-6206 (2014).
200. Chapman, B. et al. Changing epidemiology of candidaemia in Australia. J. Antimicrob. Chemother 72, 1103-1108 (2017)

201. Doi, A. M. et al. Epidemiology and microbiologic characterization of nosocomial Candidemia from a Brazilian national surveillance program. PLOS ONE 11, e0146909 (2016).

202. St-Germain, G. et al. Epidemiology and antifungal susceptibility of bloodstream Candida isolates in Quebec: report on 453 cases between 2003 and 2005 . Can. J. Infect. Dis. Med. Microbiol. 19, 55-62 (2008).

203. Astvad, K. M. T. et al. Update from a 12 -year nationwide fungemia surveillance: increasing intrinsic and acquired resistance causes concern. J. Clin. Microbiol. 56, e01564-17 (2018).

204. Lortholary, O. et al. The risk and clinical outcome of candidemia depending on underlying malignancy. Intensive Care Med. 43, 652-662 (2017).

205. Morii, D. et al. Distribution of Candida species isolated from blood cultures in hospitals in Osaka, Japan. J. Infect. Chemother 20, 558-562 (2014).

206. Cheng, S. C. et al. mTOR- and HIF-1 alpha-mediated aerobic glycolysis as metabolic basis for trained immunity. Science 345, 1250684 (2014).

207. Wirnsberger, G. et al. Inhibition of CBLB protects from lethal Candida albicans sepsis. Nat. Med. 22. 915-923 (2016).

208. Dominquez-Andres, J. et al. Inflammatory Ly6Chigh monocytes protect against candidiasis through IL-15-driven NK cell/neutrophil activation. Immunity 46, 1059-1072 (2017).

209. Collar, A. L. et al. The homozygous CX3CR1-M280 mutation impairs human monocyte survival. JCl Insight 3, 95417 (2018)

210. Zhao, X. et al. JNK1 negatively controls antifungal innate immunity by suppressing CD23 expression. Nat. Med. 23, 337-346 (2017).

211. Astvad, K. M. et al. Evaluation of caspofungin susceptibility testing by the new Vitek 2 AST-YS06 yeast card using a unique collection of FKS wild-type and hot spot mutant isolates, including the five most common candida species. Antimicrob. Agents Chemother. 57, 177-182 (2013).

\section{Acknowledgements}

This work was supported in part by the Division of Intramural Research, National Institute of Allergy and Infectious Diseases, US National Institutes of Health.

\section{Author contribution}

Introduction (P.G.P.); Epidemiology (M.C.A. and L.O.-Z.); Mechanisms/pathophysiology (M.S.L. and M.C.A.); Diagnosis screening and prevention (M.C.A.); Management (P.G.P. and B.J.K.); Quality of life (P.G.P.); Outlook (B.J.K.); Overview of Primer (P.G.P.)

\section{Competing interests}

P.G.P. has received research grants from Merck, Gilead, Astellas, Cidara, IMMY Diagnostics, T2 Biosystems and Mayne Pharma and is a scientific adviser for Cidara, IMMY, Matinas, Amplyx and Mayne Pharma. He is not affiliated with any speakers' bureau and owns no equities in pharmaceutical companies. M.C.A. has received research grants, payment for contract work on minimum inhibitory concentrations and/or ecture honoraria from the following companies: Merck, Astellas, Basilea, Pfizer, Gilead, Cidara, Amplyx, F2C Novabiotics and T2Biosystems. She is not affiliated with any speakers' bureau and owns no equities in pharmaceutical companies. L.O.-Z. has received research grants, consulting honoraria and/or lecture honoraria from the following companies: Merck, Astellas, Pfizer, Gilead, Scynexis, Cidara, Amplyx and Vical. B.J.K. is a scientific adviser for Amplyx Astellas, Basilea, Cidara and Scynexis. He is not affiliated with any speakers' bureau and owns no equities in pharmaceutical companies. M.S.L. declares no competing interests.

\section{Publisher's note}

Springer Nature remains neutral with regard to jurisdictional claims in published maps and institutional affiliations.

\section{Reviewer information}

Nature Reviews Disease Primers would like to thank C. Gil, A. Groll, G. Quindōs, M. Sanguinetti and C. Viscoli and anonymous reviewer(s) for their contribution to the peer review of this work.

RELATED LINKS

Centers for Disease Control and Prevention: https://www.
cdc.gov/fungal/diseases/candidiasis/tracking-c-auris.html 